Supplement of Hydrol. Earth Syst. Sci., 22, 3213-3227, 2018

https://doi.org/10.5194/hess-22-3213-2018-supplement

(C) Author(s) 2018. This work is distributed under

the Creative Commons Attribution 4.0 License.

(c) (1)

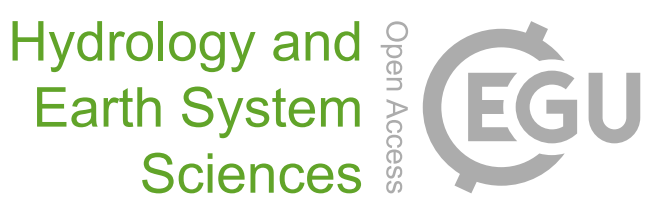

Supplement of

\title{
A risk assessment methodology to evaluate the risk failure of managed aquifer recharge in the Mediterranean Basin
}

Paula Rodríguez-Escales et al.

Correspondence to: Paula Rodríguez-Escales (paula.rodriguez.escales@upc.edu)

The copyright of individual parts of the supplement might differ from the CC BY 4.0 License. 
S1. LITERARURE REVIEW OF MANAGED AQUIFER RECHARGE FAILURES 


\begin{tabular}{|c|c|c|c|c|c|}
\hline CASE & $\begin{array}{l}\text { RECHAR } \\
\text { GE TYPE }\end{array}$ & $\begin{array}{l}\text { PLACE \& } \\
\text { TIME }\end{array}$ & $\begin{array}{l}\text { DURATIO } \\
\text { N OF THE } \\
\text { PROJECT }\end{array}$ & MAIN PROBLEMS & $\begin{array}{l}\text { REFEREN } \\
\text { CE }\end{array}$ \\
\hline $\begin{array}{l}\text { WATER FACTORY } 21 \\
\text { COASTAL BARRIER } \\
\text { SEAWATER INTRUSION }\end{array}$ & $\begin{array}{l}\text { DEEP } \\
\text { WELLS }\end{array}$ & $\begin{array}{l}\text { ORANGE } \\
\text { COUNTY } \\
\text { (USA), } \\
1977\end{array}$ & 4 YEARS & $\begin{array}{c}\text { PROBLEMS - MICROBIOLOGICAL, METEOROLOGYCAL (EXCESS OF RAIN } \\
\text { DIMINISHES THE AMOUNT OF WATER INJECTED), DESIGN AND } \\
\text { CONSTRUCTION COSTS (16 MILLION \$), OPERATION COSTS (2 MILION } \\
\text { \$ A YEAR), CLOGGING, WASTEWATER TREATMENT PLANT FAILURE, } \\
\text { SALINITY/SODICITY (WATER INTRUSION), GEOLOGIGAL } \\
\text { HETEROGENEICITY (DIFFERENT GEOLOGICAL MATERIAL LAYERS } \\
\text { PRESENT), NOT SHALLOW AQUIFER OR HIGH THICKNESS (THE WELLS } \\
\text { ARE REALLY DEEP } 850 \text { - } 1150 \text { FEET) }\end{array}$ & $\begin{array}{l}\text { (ASANO, } \\
1985)\end{array}$ \\
\hline $\begin{array}{c}\text { OPERATIONS AT THE } \\
\text { CEDAR } \\
\text { CREEK WASTEWATER } \\
\text { RECLAMATION- } \\
\text { RECHARGE } \\
\text { FACILITIES }\end{array}$ & $\begin{array}{c}\text { DEEP } \\
\text { WELLS }\end{array}$ & $\begin{array}{l}\text { NASSAU } \\
\text { COUNTY, } \\
\text { NEW } \\
\text { YORK, } \\
(1979)\end{array}$ & $\begin{array}{c}3 \text { YEARS } \\
(4 \\
\text { INCLUDIN } \\
\text { G } \\
\text { CONSTRU } \\
\text { CTION) }\end{array}$ & $\begin{array}{c}\text { PROBLEMS - DESIGN AND CONSTRUCTION COSTS ( } 22 \text { MILLION } \\
\text { DOLLARS), CIVIL WORK FAILURES "VERY LIKELY" (OTHERS - } \\
\text { UNDERDRAIN SYSTEMS, DUAL-MEDIA FILTER SYSTEM, CARBON } \\
\text { ADSORVERS, MECHANICAL/ELECTRONIC PROBLEMS), OPERATIONAL } \\
\text { COSTS (8 MILLION DOLARS), WASTEWATER TREATMENT PLANT FAILURE }\end{array}$ & $\begin{array}{c}\text { (ASANO, } \\
1985)\end{array}$ \\
\hline $\begin{array}{l}\text { PROPOSED } \\
\text { GROUNDWATER } \\
\text { RECHARGE }\end{array}$ & $\begin{array}{l}\text { DEEP } \\
\text { WELLS }\end{array}$ & $\begin{array}{l}\text { EL PASO, } \\
\text { TEXAS } \\
(1985)\end{array}$ & $\begin{array}{l}\text { UNKNOW } \\
\mathrm{N}\end{array}$ & $\begin{array}{c}\text { PROBLEMS - CONSTRUCTION COST (OVER } 22 \text { MILLION DOLLARS), } \\
\text { NUTRIENTS (NITROGEN AND PHOSPORUS), SALINITY AND SODICITY, } \\
\text { WASTEWATER TREATMENT PLANT FAILURE, SUSPENDED SOLIDS, GAS } \\
\text { GENERATION (PHYSICIAL MOTIVES AND BAD DESIGN) }\end{array}$ & $\begin{array}{l}\text { (ASANO, } \\
1985)\end{array}$ \\
\hline $\begin{array}{l}\text { GROUNDWATER } \\
\text { RECHARGE FOR } \\
\text { WASTEWATER REUSE IN } \\
\text { THE } \\
\text { DAN REGION PROJECT }\end{array}$ & $\begin{array}{l}\text { INFILTRA } \\
\text { TION } \\
\text { BASINS / } \\
\text { SPREADI } \\
\text { NG } \\
\text { BASINS }\end{array}$ & $\begin{array}{c}\text { ISRAEL, } \\
(1977)\end{array}$ & 5 YEARS & $\begin{array}{c}\text { PROBLEMS - LAND USE ( } 30 \text { HA), LOW INFILTRATION RATES, CLIMATIC } \\
\text { CONDITIONS, AND THE FREQUENCY OF BASIN CLEANING, SALINITY, } \\
\text { NUTRIENTS (N AND P HIGHER IN WINTER), SUSPENDED SOLIDS } \\
\text { (HIGHER IN WINTER), ORGANANIC CHEMICAL COMPOUNDS, } \\
\text { WASTEWATER TREATMENT PLANT FAILURE, GEOLOGIGAL } \\
\text { HETEROGENEICITY (DIFFERENT GEOLOGICAL MATERIAL LAYERS } \\
\text { PRESENT), TRACE ELEMENTS (MAINLY METALS, BUT ALSO MANGANESE } \\
\text { AND POTASSIUM) }\end{array}$ & $\begin{array}{c}\text { (ASANO, } \\
1985)\end{array}$ \\
\hline $\begin{array}{l}\text { SOIL DEPOSITION OF } \\
\text { TRACE } \\
\text { METALS DURING } \\
\text { GROUNDWATER } \\
\text { RECHARGE USING } \\
\text { SURFACE } \\
\text { SPREADING } \\
\end{array}$ & $\begin{array}{l}\text { SURFACE } \\
\text { SPREADI } \\
\quad \text { NG }\end{array}$ & $\begin{array}{l}\text { CALIFORN } \\
\text { IA (USA) }\end{array}$ & 20 YEARS & $\begin{array}{l}\text { PROBLEMS - SALINITY AND SODICITY, SUSPENDED SOLIDS, TRACE } \\
\text { ELEMENTS (OTHERS BUT MAINLY METALS), CLOGGING (NOT } \\
\text { SPECIFIED), ORGANIC CHEMICALS, WATER SCARCITY (CLIMATE) }\end{array}$ & $\begin{array}{l}\text { (ASANO, } \\
1985)\end{array}$ \\
\hline $\begin{array}{c}\text { ISSUES IN ARTIFICIAL } \\
\text { RECHARGE }\end{array}$ & GENERAL & NA & NA & $\begin{array}{l}\text { PROBLEMS - LONG TIME, CHEMICAL QUALITY ISSUES } \\
\text { NOT A PROBLEM - HAS GOOD SOCIAL ACCEPTANCE }\end{array}$ & $\begin{array}{l}\text { (BOUWER } \\
, 1996)\end{array}$ \\
\hline
\end{tabular}




\begin{tabular}{|c|c|c|c|c|c|}
\hline CASE & $\begin{array}{l}\text { RECHAR } \\
\text { GE TYPE }\end{array}$ & $\begin{array}{l}\text { PLACE \& } \\
\text { TIME }\end{array}$ & $\begin{array}{l}\text { DURATIO } \\
\text { N OF THE } \\
\text { PROJECT }\end{array}$ & MAIN PROBLEMS & $\begin{array}{l}\text { REFEREN } \\
\text { CE }\end{array}$ \\
\hline $\begin{array}{c}\text { ISSUES IN ARTIFICIAL } \\
\text { RECHARGE }\end{array}$ & $\begin{array}{l}\text { INFILTRA } \\
\text { TION } \\
\text { BASINS }\end{array}$ & NA & NA & $\begin{array}{c}\text { PROBLEMS - LAND USE, WATER QUALITY, CLOGGING, SUSPENDED } \\
\text { SOLIDS CONTENT, ORGANIC COMPOUNDS, FLOODING, DRYING, } \\
\text { NUTRIENTS (NITROGEN MAINY), BAD SOIL INFILTRATION RATE AND } \\
\text { COMPACTION }\end{array}$ & $\begin{array}{l}\text { (BOUWER } \\
, 1996)\end{array}$ \\
\hline $\begin{array}{c}\text { ISSUES IN ARTIFICIAL } \\
\text { RECHARGE }\end{array}$ & $\begin{array}{c}\text { DEEP } \\
\text { WELLS }\end{array}$ & NA & NA & $\begin{array}{c}\text { PROBLEMS - MAIN PROBLEM IS CLOGGING, SUSPENDED SOLIDS, } \\
\text { MICROORGANISMS, NUTRIENTS (N AND P), DESIGN AND } \\
\text { CONSTRUCTION COSTS, CORROSION } \\
\text { NOT A PROBLEM - CAN BE DONE IN ZONES WHERE PERMEABLE SOILS } \\
\text { ARE NOT AVAILABLE }\end{array}$ & $\begin{array}{l}\text { (BOUWER } \\
, 1996)\end{array}$ \\
\hline $\begin{array}{c}\text { ISSUES IN ARTIFICIAL } \\
\text { RECHARGE }\end{array}$ & $\begin{array}{l}\text { VADOSE } \\
\text { ZONE } \\
\text { WELLS }\end{array}$ & NA & NA & $\begin{array}{c}\text { PROBLEMS - CLOGGING, SUSPENDED SOLIDS, NUTRIENTS, } \\
\text { MICROORGANISMS, ORGANIC COMPOUNDS, LOW INFILTRATION RATES } \\
\text { NOT A PROBLEM - CHEAPER }\end{array}$ & $\begin{array}{l}\text { (BOUWER } \\
, 1996)\end{array}$ \\
\hline $\begin{array}{c}\text { ISSUES IN ARTIFICIAL } \\
\text { RECHARGE }\end{array}$ & $\begin{array}{l}\text { SEEPAGE } \\
\text { TRENCHE } \\
\quad \mathrm{S}\end{array}$ & NA & NA & $\begin{array}{c}\text { PROBLEMS - SUSPENDED SOLIDS ARE USUALLY A PROBLEM, } \\
\text { NOT A PROBLEM - CHEAPER }\end{array}$ & $\begin{array}{c}\text { (BOUWER } \\
, 1996)\end{array}$ \\
\hline $\begin{array}{l}\text { ARTIFICIAL RECHARGE } \\
\text { OF GROUNDWATER: } \\
\text { HYDROGEOLOGY AND } \\
\text { ENGINEERING }\end{array}$ & $\begin{array}{l}\text { SURFACE } \\
\text { INFILTRA } \\
\quad \text { TION }\end{array}$ & NA & NA & $\begin{array}{c}\text { PROBLEMS - FLOOD DANGER, CIVIL WORK FAILURES (OTHERS AND } \\
\text { SLOPE), LAND USE, WATER QUALITY PROBLEMS, SUSPENDED SOLIDS, } \\
\text { CLOGGING (BIOLOGICAL, MINERAL AND SEDIMENTAL), GAS } \\
\text { FORMATION (BACTERIAL MAINLY BUT THER TYPES ARE ALSO QUITE } \\
\text { TYPICAL), NUTRIENTS, ORGANIC COMPOUNDS, RISK OF LOW } \\
\text { INFILTRATION RATE, CONTAMINANT SPREADING }\end{array}$ & $\begin{array}{l}\text { (BOUWER } \\
\text {, 2002A) }\end{array}$ \\
\hline $\begin{array}{l}\text { ARTIFICIAL RECHARGE } \\
\text { OF GROUNDWATER: } \\
\text { HYDROGEOLOGY AND } \\
\text { ENGINEERING }\end{array}$ & $\begin{array}{l}\text { VADOSE- } \\
\text { ZONE } \\
\text { INFILTRA } \\
\quad \text { TION }\end{array}$ & NA & NA & $\begin{array}{c}\text { PROBLEMS - VERY LIKELY RISK OF INSUFICIENT SOIL INFILTRATION } \\
\text { RATE, LAND USE, PIPELINE FAILURE, GAS ACCUMULATION (PHYSICAL), } \\
\text { PIPE FAILURE, MAINLY DISADVANTAGE IS CLOGGING (BIOLOGICAL AND } \\
\text { SEDIMENTAL), SUSPENDED SOLIDS CONTENT, } \\
\text { NOT PROBLEM - CHEAPER }\end{array}$ & $\begin{array}{c}\text { (BOUWER } \\
, \text { 2002A) }\end{array}$ \\
\hline $\begin{array}{l}\text { ARTIFICIAL RECHARGE } \\
\text { OF GROUNDWATER: } \\
\text { HYDROGEOLOGY AND } \\
\text { ENGINEERING }\end{array}$ & WELLS & NA & NA & $\begin{array}{c}\text { PROBLEMS - COMPACTION, CLOGGING (MOST TYPICALL PROBLEM, DUE } \\
\text { TO SEDIMENTS BUT ALSO OTHER REASONS LIKE BACTERIA OR } \\
\text { PRECIPITATION), WATER QUALITY, NUTRIENTS, SALINITY, } \\
\text { MICROBIOLOGICAL PROBLEMS, } \\
\text { NOT PROBLEM - LAND USE, INFILTRATION RATE }\end{array}$ & $\begin{array}{l}\text { (BOUWER } \\
, 2002 A)\end{array}$ \\
\hline $\begin{array}{l}\text { ARTIFICIAL RECHARGE } \\
\text { OF GROUNDWATER: } \\
\text { HYDROGEOLOGY AND } \\
\text { ENGINEERING }\end{array}$ & $\begin{array}{l}\text { GENERAL } \\
\text { ARTIFICI } \\
\text { AL } \\
\text { RECHAR }\end{array}$ & NA & NA & $\begin{array}{c}\text { THE MAIN ISSUE IN ARTIFICIAL RECHARGE IS CLOGGING, AVAILABILITY } \\
\text { OF WATER RESOURCES IS ALSO A PROBLEM WITH CLIMATIC ISSUES, } \\
\text { SOCIAL COSTS, ENVIRONMENTAL COSTS, LAND USE, CIVIL WORK } \\
\text { PROBLEMS (IN GENERAL, CORROSION), }\end{array}$ & $\begin{array}{l}\text { (BOUWER } \\
, 2002 A)\end{array}$ \\
\hline
\end{tabular}




\begin{tabular}{|c|c|c|c|c|c|}
\hline CASE & $\begin{array}{l}\text { RECHAR } \\
\text { GE TYPE }\end{array}$ & $\begin{array}{l}\text { PLACE \& } \\
\text { TIME }\end{array}$ & $\begin{array}{l}\text { DURATIO } \\
\text { N OF THE } \\
\text { PROJECT }\end{array}$ & MAIN PROBLEMS & $\begin{array}{l}\text { REFEREN } \\
\text { CE }\end{array}$ \\
\hline & $\begin{array}{c}\text { GE } \\
\text { SYSTEMS }\end{array}$ & & & & \\
\hline $\begin{array}{c}\text { ARTIFICIAL RECHARGE } \\
\text { OF AQUIFERS }\end{array}$ & $\begin{array}{l}\text { INFILTRA } \\
\text { TION } \\
\text { BASINS } \\
\text { AND } \\
\text { CANALS }\end{array}$ & $\begin{array}{l}\text { SAN JUAN } \\
\text { RIVER } \\
\text { BASIN } \\
\text { (ARGENTI } \\
\text { NA) }\end{array}$ & NA & $\begin{array}{l}\text { PROBLEMS - SEDIMENTATION OF FINE MATERIAL (CLOGGING, } \\
\text { TURBIDITY, ETC.), FLOODING RISK (THE FLOODS MAY INTERFERE WITH } \\
\text { THE INFILTRATION BASIN), DEPOSITION PROBLEMS, CORROSION, } \\
\text { EROSION PROBLEMS, CIVIL DAMAGE (OTHERS), VANDALISM PROBLEMS } \\
\text { (MAY BE CONSIDERED AS TERRORISM?), DROUGH PROBLEMS, DURING } \\
\text { DROUGH STAGES THERE MAY BE A SHORTAGE OF WATER RESOURCES } \\
\text { TO FEED THE RECHARGE, LACK OF INCENTIVES (LEGISLATIVE AND } \\
\text { ECONOMICAL) FOR MAINTENANCE, LACK OF ENOUGH WATER TO HAVE } \\
\text { AN ECONOMICALLY FEASIBLE RECHARGE, PROBLEM WITH NUTRIENTS } \\
\text { (N AND P), RISK OF AQUIFER DISSOLUTION, LEGISLATION PROBLEMS } \\
\text { (OTHERS, RELATED TO ENVIRONMENTAL IMPACT OF THE LANDSCAPE), } \\
\text { HIGH THICKNESS AND NOT SHALLOW AQUIFER } \\
\text { NOT PROBLEM - LOW MAINTENANCE COSTS, LOW DESIGN AND } \\
\text { CONSTRUCTION COSTS, USUALY THE TECHNICAL KNOWLEDGE OF } \\
\text { THIS TECHNOLOGY IS HIGH, }\end{array}$ & $\begin{array}{l}\text { (NATIONS } \\
\text { AND } \\
\text { PROGRAM } \\
\text { ME, } \\
\text { 2011)) }\end{array}$ \\
\hline $\begin{array}{l}\text { INVESTIGATING THE } \\
\text { CAUSES OF WATER- } \\
\text { WELL FAILURE IN THE } \\
\text { GAOTLHOBOGWE } \\
\text { WELLFIELD }\end{array}$ & $\begin{array}{l}\text { DEEP } \\
\text { WELLS }\end{array}$ & $\begin{array}{l}\text { SOUTHEA } \\
\text { ST } \\
\text { BOTSWAN } \\
\text { A }\end{array}$ & 8 YEARS & $\begin{array}{c}\text { PROBLEMS - WATER QUALITY (METALS, SALINITY/SODICITY, } \\
\text { NITROGEN, PHOSPHORUS, ETC.), LOW QUANTITY OF WATER } \\
\text { RESOURCES, PROBLEMS WITH INFILTRATION RATE, RISK OF LOW } \\
\text { WATER STORAGE, CHEMICAL CLOGGING (PRECIPITATION OF CALCITE } \\
\text { DUE TO WATER MIXTURE), PROBLEMS WITH THE DESIGN AND } \\
\text { OPERATION OF THE WELLS }\end{array}$ & $\begin{array}{l}\text { (CHAOKA } \\
\text { ET AL., } \\
\text { 2006) }\end{array}$ \\
\hline $\begin{array}{c}\text { AQUIFER STORAGE AND } \\
\text { RECOVERY }\end{array}$ & $\begin{array}{l}\text { DEEP } \\
\text { WELLS }\end{array}$ & $\begin{array}{l}\text { CALIFORN } \\
\text { IA (USA) }\end{array}$ & NA & $\begin{array}{c}\text { PROBLEMS - WATER QUALITY (SUSPENDED SOLIDS, } \\
\text { SALINITY/SODICITY, SOCIAL UNACCEPTANCE (TASTE IN WATER), LEGAL } \\
\text { CONSTRAINTS (NOT ACCOMPLISHING DRINKING STANDARDS), } \\
\text { MOVILISATION OF TRACE ELEMENTS, PRECIPITATION (CHEMICAL } \\
\text { CLOGGING), CLOGGING (SEDIMENT AND MICROBIOLOGICAL), IN } \\
\text { GENERAL CLOGGING IS THE MOST TYPICALL PROBLEM, INFILTRATION } \\
\text { PROBLEMS, CIVIL WORK FAILURES (LIQUEFACTION), NATURAL } \\
\text { HAZARDS (EARTHOUAKE), TERRORISTS ATTACKS }\end{array}$ & $\begin{array}{l}\text { ("USGS } \\
\text { CALIFORN } \\
\text { IA WATER } \\
\text { SCIENCE } \\
\text { CENTER," } \\
\text { 2015) }\end{array}$ \\
\hline $\begin{array}{c}\text { TROUBLESHOOTING } \\
\text { WATER WELL } \\
\text { PROBLEMS }\end{array}$ & $\begin{array}{l}\text { DEEP } \\
\text { WELLS }\end{array}$ & NA & NA & $\begin{array}{l}\text { PROBLEMS - IMPROPER WELL DESIGN AND OPERATION, INCOMPLETE } \\
\text { WELL DEVELOPMENT, BOREHOLE STABILITY PROBLEMS, INCRUSTATION } \\
\text { BUILD-UP (CLOGGING DUE TO CHEMICAL ISSUES WITH WATER), } \\
\text { BIOFOULING CLOGGING DUE TO MICROBIOLOGICAL ISSUES), } \\
\text { CORROSION, AQUIFER PROBLEMS, OVER PUMPING (SEDIMENT } \\
\text { PARTICLE MOVING, SEDIMENTATION, EROSION, COMPACTION), } \\
\text { NUTRIENT PROBLEMS (N AND P), GAS GENERATION (BACTERIAL AND }\end{array}$ & $\begin{array}{l}\text { (GOVERN } \\
\text { MENT OF } \\
\text { ALBERTA, } \\
\text { N.D.) }\end{array}$ \\
\hline
\end{tabular}




\begin{tabular}{|c|c|c|c|c|c|}
\hline CASE & $\begin{array}{l}\text { RECHAR } \\
\text { GE TYPE }\end{array}$ & $\begin{array}{l}\text { PLACE \& } \\
\text { TIME }\end{array}$ & $\begin{array}{l}\text { DURATIO } \\
\text { N OF THE } \\
\text { PROJECT }\end{array}$ & MAIN PROBLEMS & $\begin{array}{c}\text { REFEREN } \\
\text { CE }\end{array}$ \\
\hline & & & & $\begin{array}{c}\text { INAPROPIATE DESIGN), LACK OF RECHARGE, CLIMATE ISSUES, } \\
\text { DROUGH PERIODS, CIVIL WORK FAILURE (PIPES BREAKAGE AND } \\
\text { OTHERS), LOW INFILTRATION, WATER QUALITY ISSUES (METALS, } \\
\text { NUTRIENTS AND ORGANIC COMPOUNDS) }\end{array}$ & \\
\hline $\begin{array}{l}\text { AUSTRALIAN } \\
\text { GUIDELINES FOR WATER } \\
\text { RECYCLING: MANAGED } \\
\text { AQUIFER RECHARGE }\end{array}$ & $\begin{array}{l}\text { DEEP } \\
\text { WELLS }\end{array}$ & $\begin{array}{c}\text { AUSTRALI } \\
\text { A }\end{array}$ & NA & $\begin{array}{l}\text { NOT PROBLEM - LOW CAPITAL COSTS (MANAGED RECHARGE IS OFTEN } \\
\text { THE MOST ECONOMIC FORM OF NEW WATER SUPPLY), NO } \\
\text { EVAPORATION LOSS, NOT ALGAE OR MOSQUITOES (UNLIKE DAMS), NO } \\
\text { LOSS OF PRIME VALLEY FLOOR LAND (EROSION), ABILITY TO USE } \\
\text { SALINE AQUIFERS THAT COULD NOT BE DIRECTLY USED FOR SUPPLIES, } \\
\text { POTENTIAL LOCATION CLOSE TO NEW WATER SOURCES, AND WHERE } \\
\text { DEMAND FOR WATER IS HIGH, AQUIFERS PROVIDING TREATMENT AS } \\
\text { WELL AS STORAGE, LOW GREENHOUSE GAS EMISSIONS COMPARED TO } \\
\text { REMOTE PUMPED STORAGES, ABLE TO BE BUILT TO THE SIZE } \\
\text { REQUIRED FOR INCREMENTAL GROWTH IN WATER DEMAND, PROVISION } \\
\text { OF EMERGENCY AND STRATEGIC RESERVES, IMPROVED RELIABILITY OF } \\
\text { EXISTING SUPPLIES, IMPROVED ENVIRONMENTAL FLOWS IN WATER } \\
\text { SUPPLY CATCHMENTS FOR URBAN AREAS }\end{array}$ & $\begin{array}{l}(\mathrm{EPHC} / \mathrm{NH} \\
\mathrm{MRC} / \mathrm{NRM} \\
\mathrm{MC}, 2008)\end{array}$ \\
\hline $\begin{array}{l}\text { AUSTRALIAN } \\
\text { GUIDELINES FOR WATER } \\
\text { RECYCLING: MANAGED } \\
\text { AQUIFER RECHARGE }\end{array}$ & $\begin{array}{l}\text { GENERAL } \\
\text { ARTIFICI } \\
\text { AL } \\
\text { RECHAR } \\
\text { GE } \\
\text { SYSTEMS }\end{array}$ & $\begin{array}{c}\text { AUSTRALI } \\
\text { A }\end{array}$ & NA & $\begin{array}{c}\text { DEEP WELLS - PREFERABLY USED WHEN THERE ARE CONFINED } \\
\text { AQUIFERS OR SUPERFICIAL CLAY LEVELS, CAN WORK WITH LOW } \\
\text { INFILTRATION RATE, LOW LAND USE/COST, EASE OF TRAFFIC ACCESS, } \\
\text { COMPATIBILITY OF LAND USE, SUSPENDED SOLIDS AND NUTRIENTS } \\
\text { USUALY LEAD TO CLOGGING PROBLEMS } \\
\text { INFILTRATION PONDS - PREFERED WHEN LAND COST/USE IS CHEAP } \\
\text { GENERAL INFO - USUALLY ARTIFICIAL RECHARGE HAS GOOD SOCIAL } \\
\text { ACCEPTANCE AND SUFICIENT RESIDENCE TIMES FOR WATER, THIS } \\
\text { RESIDENCE TIME IMPLIES LESS TREATMENT FOR THE WATER AND LESS } \\
\text { RISK FOR PATHOGENS }\end{array}$ & $\begin{array}{l}\text { (EPHC/NH } \\
\text { MRC/NRM } \\
\text { MC, 2008) }\end{array}$ \\
\hline $\begin{array}{l}\text { AUSTRALIAN } \\
\text { GUIDELINES FOR WATER } \\
\text { RECYCLING: MANAGED } \\
\text { AQUIFER RECHARGE }\end{array}$ & $\begin{array}{l}\text { GENERAL } \\
\text { ARTIFICI } \\
\quad \text { AL } \\
\text { RECHAR } \\
\quad \text { GE } \\
\text { SYSTEMS }\end{array}$ & $\begin{array}{c}\text { AUSTRALI } \\
\text { A }\end{array}$ & NA & $\begin{array}{c}\text { GENERAL INFO - ARTIFIAL RECHARGE DEPENDS MAINLY ON THE } \\
\text { AVAILABILITY OF APROPIATE AQUIFERS, SUFFICIENT VOLUMES OF } \\
\text { WATER ARE NEEDED TO JUSTIFY THE COSTS OF THE PROJECT, PLACES } \\
\text { WITH SURFACE AQUIFERS CAUSE STRUCTURAL PROBLEMS, } \\
\text { SALINISATION AND WATERLOGGING. }\end{array}$ & $\begin{array}{l}(\mathrm{EPHC} / \mathrm{NH} \\
\mathrm{MRC} / \mathrm{NRM} \\
\mathrm{MC}, 2008)\end{array}$ \\
\hline $\begin{array}{l}\text { AUSTRALIAN } \\
\text { GUIDELINES FOR WATER } \\
\text { RECYCLING: MANAGED } \\
\text { AQUIFER RECHARGE }\end{array}$ & $\begin{array}{c}\text { DEEP } \\
\text { WELLS }\end{array}$ & $\begin{array}{l}\text { NORTHER } \\
N \\
\text { ADELAIDE } \\
\text { PLAINS } \\
\text { (AUSTRAL } \\
\text { IA) }\end{array}$ & NA & $\begin{array}{c}\text { PROBLEMS - SALINITY, AQUIFER HETEROGENITY, WATER MIXTURE, } \\
\text { NEED TO HAVE A WATER TREATMENT PLANT (DESIGN AND } \\
\text { CONSTRUCTION COSTS, OPERATIONAL COSTS) } \\
\text { NOT PROBLEMS - MEET DRINKIG WATER REQUERIMENTS }\end{array}$ & $\begin{array}{l}\text { (EPHC/NH } \\
\text { MRC/NRM } \\
\text { MC, 2008) }\end{array}$ \\
\hline
\end{tabular}




\begin{tabular}{|c|c|c|c|c|c|}
\hline CASE & $\begin{array}{l}\text { RECHAR } \\
\text { GE TYPE }\end{array}$ & $\begin{array}{l}\text { PLACE \& } \\
\text { TIME }\end{array}$ & $\begin{array}{l}\text { DURATIO } \\
\text { N OF THE } \\
\text { PROJECT }\end{array}$ & MAIN PROBLEMS & $\begin{array}{l}\text { REFEREN } \\
\text { CE }\end{array}$ \\
\hline $\begin{array}{l}\text { AUSTRALIAN } \\
\text { GUIDELINES FOR WATER } \\
\text { RECYCLING: MANAGED } \\
\text { AQUIFER RECHARGE }\end{array}$ & $\begin{array}{l}\text { GENERAL } \\
\text { ARTIFICI } \\
\text { AL } \\
\text { RECHAR } \\
\text { GE } \\
\text { SYSTEMS }\end{array}$ & $\begin{array}{c}\text { AUSTRALI } \\
\text { A }\end{array}$ & NA & $\begin{array}{l}\text { PROBLEMS - PATHOGENS, INORGANIC CHEMICALS, SALINITY AND } \\
\text { SODICITY, NUTRIENTS, ORGANIC CHEMICALS, TURBIDITY AND } \\
\text { PARTICULATES, RADIONUCLIDES, PRESSURE/FLOW } \\
\text { RATES/VOLUMES/LEVELS OF WATER, CONTAMINANT MIGRATION IN } \\
\text { FRACTURED AND CARSTIC AQUIFERS, AQUIFER DISSOLUTION, WELL } \\
\text { STABILITY, IMPACT ON GROUNDWATER ECOSYSTEMS, GREENHOUSE } \\
\text { GASES GENERATION (MICROBIOLOGICAL ISSUES) }\end{array}$ & $\begin{array}{l}(\mathrm{EPHC} / \mathrm{NH} \\
\mathrm{MRC} / \mathrm{NRM} \\
\mathrm{MC}, 2008)\end{array}$ \\
\hline $\begin{array}{l}\text { AUSTRALIAN } \\
\text { GUIDELINES FOR WATER } \\
\text { RECYCLING: MANAGED } \\
\text { AQUIFER RECHARGE }\end{array}$ & $\begin{array}{l}\text { GENERAL } \\
\text { ARTIFICI } \\
\text { AL } \\
\text { RECHAR } \\
\text { GE } \\
\text { SYSTEMS }\end{array}$ & $\begin{array}{c}\text { AUSTRALI } \\
\text { A }\end{array}$ & NA & $\begin{array}{l}\text { PROBLEMS - INCREASE IRON, MANGANESE, ARSENIC, TRACE SPECIES } \\
\text { AND HYDROGEN SULFIDE, SODICITY/SALINITY PROBEMS, NTRIENT } \\
\text { ISSUES, }\end{array}$ & $\begin{array}{l}(\mathrm{EPHC} / \mathrm{NH} \\
\mathrm{MRC} / \mathrm{NRM} \\
\mathrm{MC}, 2008)\end{array}$ \\
\hline $\begin{array}{l}\text { MOBILIZATION OF } \\
\text { ARSENIC AND OTHER } \\
\text { TRACE ELEMENTS } \\
\text { DURING AQUIFER } \\
\text { STORAGE AND } \\
\text { RECOVERY, SOUTHWEST } \\
\text { FLORIDA }\end{array}$ & $\begin{array}{c}\text { DEEP } \\
\text { WELLS }\end{array}$ & $\begin{array}{c}\text { FLORIDA } \\
\text { (USA) }\end{array}$ & NA & $\begin{array}{c}\text { PROBLEMS - ARSENIC, MANGANESE, URANIUM (RADIONUCLIDES), } \\
\text { ORGANIC COMPOUNDS, WATER RESIDENCE TIME, AQUIFER AND INPUT } \\
\text { WATER CHEMISTRY PROBLEMS (DO, PH, ETC.), AQUIFER MATRIX } \\
\text { CHEMISTRY/MINERALOGY, SITE SPECIFIC } \\
\text { HIDROGEOLOGY/HIDROCHEMISTRY, WATER MIXTURE }\end{array}$ & $\begin{array}{l}\text { (AIKEN } \\
\text { AND } \\
\text { KUNIANS } \\
\text { KY, 2002) }\end{array}$ \\
\hline $\begin{array}{l}\text { AUSTRALIAN } \\
\text { GUIDELINES FOR WATER } \\
\text { RECYCLING: MANAGED } \\
\text { AQUIFER RECHARGE }\end{array}$ & $\begin{array}{l}\text { GENERAL } \\
\text { ARTIFICI } \\
\text { AL } \\
\text { RECHAR } \\
\text { GE } \\
\text { SYSTEMS }\end{array}$ & $\begin{array}{c}\text { AUSTRALI } \\
\text { A }\end{array}$ & NA & $\begin{array}{c}\text { GENERAL INFO - ABOUT CLOGGING THERE'S INFO FROM } 14 \text { INJECTION } \\
\text { PLACES THAT SUFERED CLOGGING PROBLEMS. FROM THE } 14 \text { SITES, } 8 \\
\text { WERE BIOLOGICAL CLOGGING, } 9 \text { PHYSICAL CLOGGING AND } 1 \text { WAS } \\
\text { CHEMICAL CLOGGING (IN SOME CASES THERE WAS A MIXTURE } \\
\text { BETWEEN TWO TYUPES OF CLOGGING) }\end{array}$ & $\begin{array}{l}(\mathrm{EPHC} / \mathrm{NH} \\
\mathrm{MRC} / \mathrm{NRM} \\
\mathrm{MC}, 2008)\end{array}$ \\
\hline $\begin{array}{c}\text { SOURCES OF HIGH- } \\
\text { CHLORIDE WATER TO } \\
\text { WELLS, EASTERN SAN } \\
\text { JOAQUIN GROUND- } \\
\text { WATER SUBBASIN, } \\
\text { CALIFORNIA }\end{array}$ & $\begin{array}{c}\text { DEEP } \\
\text { WELLS }\end{array}$ & $\begin{array}{l}\text { CALIFORN } \\
\text { IA (USA) }\end{array}$ & NA & $\begin{array}{c}\text { PROBLEMS - SALINITY/SODICITY, CHLORIDE, METALS (ARSENIC, } \\
\text { MANGANESE, ETC.), NUTRIENTS (NITRATES), WATER MIXTURE, WATER } \\
\text { EVAPORATION }\end{array}$ & $\begin{array}{l}\text { (AIKEN } \\
\text { AND } \\
\text { KUNIANS } \\
\text { KY, 2002) }\end{array}$ \\
\hline $\begin{array}{l}\text { AQUIFER STORAGE AND } \\
\text { RECOVERY FOR THE } \\
\text { CITY OF ROSEVILLE: } \\
\text { A CONJUNCTIVE USE } \\
\text { PILOT PROJECT }\end{array}$ & $\begin{array}{c}\text { DEEP } \\
\text { WELLS }\end{array}$ & $\begin{array}{l}\text { CALIFORN } \\
\text { IA (USA) }\end{array}$ & NA & $\begin{array}{c}\text { PROBLEMS - ORGANIC CHEMICALS (THM, DBP), DESIGN AND } \\
\text { CONSTRUCTION COSTS (PROJECTS OF WATER RECHARGE WITH A COST } \\
\text { OF MORE THAN } 215 \text { MILLION \$), LEGISLATION ISSUES (NATIONAL AND } \\
\text { LACK OF COORDINATION), TRACE ELEMENTS (METALS), MECHANICAL } \\
\text { COMPLICATIONS (CIVIL WORK FAILURE - OTHERS), } \\
\text { SODICITY/SALINITY, MICROBIOLOGICAL ISSUES (LEGISLATION ABOUT } \\
\text { BACTERIA INPUT IN THE RECHARGE WATER), WATER MIXTURE, }\end{array}$ & $\begin{array}{l}\text { (AIKEN } \\
\text { AND } \\
\text { KUNIANS } \\
\text { KY, 2002) }\end{array}$ \\
\hline
\end{tabular}




\begin{tabular}{|c|c|c|c|c|c|}
\hline CASE & $\begin{array}{l}\text { RECHAR } \\
\text { GE TYPE }\end{array}$ & $\begin{array}{l}\text { PLACE \& } \\
\text { TIME }\end{array}$ & $\begin{array}{l}\text { DURATIO } \\
\text { N OF THE } \\
\text { PROJECT }\end{array}$ & MAIN PROBLEMS & $\begin{array}{l}\text { REFEREN } \\
\text { CE }\end{array}$ \\
\hline & & & & $\begin{array}{l}\text { QUALITY ISSUES (IN GENERAL, IT DOESNN'T SPECIFY), AQUIFER } \\
\text { THICKNESS AND AQUIFER DEPTH, WATER SCARCITY (DROUGHT) } \\
\text { NOT PROBLEM - NATURAL ATENUATION, }\end{array}$ & \\
\hline $\begin{array}{l}\text { SAN GORGONIO PASS } \\
\text { ARTIFICIAL RECHARGE } \\
\text { INVESTIGATION }\end{array}$ & $\begin{array}{c}\text { DEEP } \\
\text { WELLS }\end{array}$ & $\begin{array}{l}\text { CALIFORN } \\
\text { IA (USA) } \\
1997\end{array}$ & 6 YEARS & $\begin{array}{c}\text { PROBLEMS - LOW INFILTRATION RATE, HIGH THICKNESS/NOT } \\
\text { SHALLOW AQUIFER, NATURAL HAZARDS (EARTHQUAKES), NUTRIENTS } \\
\text { (NITROGEN DUE TO WASTEWATER LEAKAGE) }\end{array}$ & $\begin{array}{l}(\text { FLINT } \\
\text { AND } \\
\text { ELLETT, } \\
2005)\end{array}$ \\
\hline $\begin{array}{l}\text { THE EFFECTS OF } \\
\text { ARTIFICIAL RECHARGE } \\
\text { ON GROUNDWATER } \\
\text { LEVELS } \\
\text { AND WATER QUALITY IN } \\
\text { THE WEST } \\
\text { HYDROGEOLOGIC UNIT } \\
\text { OF THE } \\
\text { WARREN SUBBASIN, } \\
\text { SAN BERNARDINO } \\
\text { COUNTY, CALIFORNIA }\end{array}$ & $\begin{array}{c}\text { DEEP } \\
\text { WELLS }\end{array}$ & $\begin{array}{c}\text { CALIFORN } \\
\text { IA (USA) } \\
2004\end{array}$ & 5 YEARS & $\begin{array}{l}\text { PROBLEMS - LOW INFILTRATION RATE, RESIDENCE TIME, LAND USE, } \\
\text { RISK OF NUTRIENT MOBILISATION, WATER LEVEL DECLINE, NUTRIENTS } \\
\text { (NITROGEN), ORGANIC CHEMICALS, WATER SCARCITY (DROUGHTS AND } \\
\text { RAINFALL PERIODICITY), EVAPORATION, SEDIMENTATION, EROSION, } \\
\text { REGIONAL HYDROGEOLOGY WATER IMBALANCE, } \\
\text { NO PROBLEM - NATURAL ATENUATION }\end{array}$ & $\begin{array}{c}\text { (U.S. } \\
\text { GEOLOGI } \\
\text { CAL } \\
\text { SURVEY, } \\
\text { 2013) }\end{array}$ \\
\hline $\begin{array}{l}\text { HYDRO-LOGIC EFFECTS } \\
\text { OF ARTIFICIAL-RE } \\
\text { CHARGE } \\
\text { EXPERIMENTS WITH } \\
\text { RECLAIMED WATER AT } \\
\text { EAST MEADOW, LONG } \\
\text { ISLAND, NEW YORK }\end{array}$ & $\begin{array}{l}\text { INFILTRA } \\
\text { TION } \\
\text { BASINS }\end{array}$ & $\begin{array}{l}\text { NEW } \\
\text { YORK } \\
(\text { USA) } \\
1982\end{array}$ & 2 YEARS & $\begin{array}{c}\text { PROBLEMS - LAND USE, GAS GENERATION (PHYSICAL MOTIVES AND } \\
\text { BAD DESIGN), LOW INFILTRATION RATES, SUSPENDED SOLIDS, } \\
\text { CLOGGING (PHYSICAL AND BIOLOGICAL), MECHANICAL FAILURES, } \\
\text { PONDING (UNWANTED WATER ACCUMULATION), MICROBIOLOGICAL } \\
\text { ISSUES, DEVELOPMENT OF INSECT POPULATIONS, WATER QUALITY } \\
\text { ISSUES (MAINLY MICROBIOLOGICAL, NUTRIENTS AND MAYBE OTHER } \\
\text { WATER CHEMICAL COMPOUNDS), SALINITY/SODICITY, METALS, SLOPE } \\
\text { FACTOR ISSUES (MOUNDING), WATER MIXING, ORGANIC CHEMICALS } \\
\text { (ORGANIC MATTER), INORGANIC CHEMICALS, INEFICIENT NATURAL } \\
\text { ATTENUATION (DUE TO SHORT RESIDENCE TIME, NOT ENOUGH } \\
\text { REACTION OF THE GEOLOGICAL MATERIALS OR DUE TO THE HIGH } \\
\text { TREATMENT OF THE INJECTED WATER) }\end{array}$ & $\begin{array}{c}\text { (SCHNEID } \\
\text { ER ET AL., } \\
1987 \text { ) }\end{array}$ \\
\hline $\begin{array}{l}\text { HYDRO-LOGIC EFFECTS } \\
\text { OF ARTIFICIAL-RE } \\
\text { CHARGE } \\
\text { EXPERIMENTS WITH } \\
\text { RECLAIMED WATER AT } \\
\text { EAST MEADOW, LONG } \\
\text { ISLAND, NEW YORK } \\
\end{array}$ & $\begin{array}{c}\text { DEEP } \\
\text { WELLS }\end{array}$ & $\begin{array}{l}\text { NEW } \\
\text { YORK } \\
(\text { USA) } \\
1982\end{array}$ & 2 YEARS & $\begin{array}{l}\text { PROBLEMS - SUSPENDED SOLIDS (TURBIDITY), CLOGGING } \\
\text { (BACTERIAL, PHYSICAL AND CHEMICAL), METALS (IRON), } \\
\text { SALINITY/SODICITY, LESS EFFICIENT TO MOVE LARGE QUANTITIES OF } \\
\text { WATER THAN THE INFILTRATION BASINS, CLOGGING IS MORE A } \\
\text { PROBLEM IN WELLS THAN IN BASINS, SLOPE FACTOR ISSUES } \\
\text { (MOUNDING), INEFICIENT NATURAL ATTENUATION (DUE TO SHORT } \\
\text { RESIDENCE TIME, NOT ENOUGH REACTION OF THE GEOLOGICAL }\end{array}$ & $\begin{array}{c}\text { (SCHNEID } \\
\text { ER ET AL., } \\
1987 \text { ) }\end{array}$ \\
\hline
\end{tabular}




\begin{tabular}{|c|c|c|c|c|c|}
\hline CASE & $\begin{array}{l}\text { RECHAR } \\
\text { GE TYPE }\end{array}$ & $\begin{array}{l}\text { PLACE \& } \\
\text { TIME }\end{array}$ & $\begin{array}{l}\text { DURATIO } \\
\text { N OF THE } \\
\text { PROJECT }\end{array}$ & MAIN PROBLEMS & $\begin{array}{l}\text { REFEREN } \\
\text { CE }\end{array}$ \\
\hline & & & & $\begin{array}{l}\text { MATERIALS OR DUE TO THE HIGH TREATMENT OF THE INJECTED } \\
\text { WATER) }\end{array}$ & \\
\hline $\begin{array}{l}\text { THE ATLANTIS WATER } \\
\text { RESOURCE } \\
\text { MANAGEMENT SCHEME: } \\
30 \text { YEARS OF ARTIFICIAL } \\
\text { GROUNDWATER } \\
\text { RECHARGE }\end{array}$ & $\begin{array}{l}\text { INFILTRA } \\
\text { TION } \\
\text { BASINS }\end{array}$ & $\begin{array}{l}\text { SOUTH } \\
\text { AFRICA } \\
(1980)\end{array}$ & 30 YEARS & $\begin{array}{c}\text { PROBLEMS - CLOGGING (PHYSICAL, BIOLOGICAL AND CHEMICAL), } \\
\text { METAL CONTENT (IRON), NOT ENOUGH WATER QUANTITY, ORGANIC } \\
\text { MATTER, LOW INFILTRATION RATE, HIGH MAINTENANCE COSTS, } \\
\text { GROUNDWATER POLLUTION, APPEREANCE OF ALIEN VEGETAL SPECIES, } \\
\text { MICROBIOLOGICAL ISSUES, LAND OWNERSHIP PROBLEMS (IS NOT } \\
\text { UNDER THE SAME LEGAL MANAGEMENT THAN THE REST OF THE } \\
\text { RECHARGE FACILITY) } \\
\text { NOT PROBLEM - LOW SALINITY }\end{array}$ & $\begin{array}{l}\text { (TREDOU } \\
\text { X AND } \\
\text { CAIN, } \\
2010)\end{array}$ \\
\hline $\begin{array}{l}\text { THE ATLANTIS WATER } \\
\text { RESOURCE } \\
\text { MANAGEMENT SCHEME: } \\
30 \text { YEARS OF ARTIFICIAL } \\
\text { GROUNDWATER } \\
\text { RECHARGE }\end{array}$ & $\begin{array}{l}\text { DEEP } \\
\text { WELLS }\end{array}$ & $\begin{array}{l}\text { SOUTH } \\
\text { AFRICA } \\
(1980)\end{array}$ & 30 YEARS & $\begin{array}{l}\text { PROBLEMS - CLOGGING (PHYSICAL, BIOLOGICAL AND CHEMICAL), } \\
\text { METAL CONTENT (IRON), NOT ENOUGH WATER QUANTITY, ORGANIC } \\
\text { MATTER, LOW INFILTRATION RATE, HIGH MAINTENANCE COSTS, } \\
\text { DROUGH CONDITIONS, OVERPUMPING WATER (IMBALANCE BETWEEN } \\
\text { THE WATER INJECTION AND PUMPING), GAS GENERATION (DUE TO } \\
\text { PHYSICAL PROPERTIES AND INEFICIENT DESIGN), GROUNDWATER } \\
\text { POLLUTION, SALINITY/SODICITY PROBLEMS, MICROBIOLOGICAL } \\
\text { ISSUES, LAND OWNERSHIP PROBLEMS (IS NOT UNDER THE SAME } \\
\text { LEGAL MANAGEMENT THAN THE REST OF THE RECHARGE FACILITY) }\end{array}$ & $\begin{array}{l}\text { (TREDOU } \\
\text { X AND } \\
\text { CAIN, } \\
2010)\end{array}$ \\
\hline $\begin{array}{c}\text { RECYCLING } \\
\text { POLOKWANE'S TREATED } \\
\text { WASTEWATER }\end{array}$ & $\begin{array}{l}\text { INFILTRA } \\
\text { TION } \\
\text { PONDS }\end{array}$ & $\begin{array}{l}\text { SOUTH } \\
\text { AFRICA }\end{array}$ & NA & $\begin{array}{c}\text { PROBLEMS - HIGH THICKNESS AND NOT SHALLOW AQUIFER, } \\
\text { EVAPORATION OF WATER (WATER LOSS), NUTRIENT PROBLEMS } \\
\text { (MAINLY NITROGEN) }\end{array}$ & $\begin{array}{l}\text { (TREDOU } \\
\text { X AND } \\
\text { CAIN, } \\
2010)\end{array}$ \\
\hline $\begin{array}{c}\text { SMALL-SCALE } \\
\text { BOREHOLE INJECTION } \\
\text { IN NAMAQUALAND }\end{array}$ & $\begin{array}{c}\text { DEEP } \\
\text { WELLS }\end{array}$ & $\begin{array}{l}\text { SOUTH } \\
\text { AFRICA } \\
(1999)\end{array}$ & 3 YEARS & $\begin{array}{l}\text { PROBLEMS - LOW INFILTRATION RATE, SALINITY/SODICITY, CLOGGING } \\
\text { (PHYSICAL) }\end{array}$ & $\begin{array}{l}\text { (TREDOU } \\
\text { X AND } \\
\text { CAIN, } \\
\text { 2010) }\end{array}$ \\
\hline $\begin{array}{l}\text { CALVINIA: TRIAL } \\
\text { BOREHOLE INJECTION } \\
\text { TESTS AND WATER } \\
\text { QUALITY ASSESSMENT } \\
\text { IN FRACTURED } \\
\text { MUDSTONES } \\
\end{array}$ & $\begin{array}{l}\text { DEEP } \\
\text { WELLS }\end{array}$ & $\begin{array}{l}\text { SOUTH } \\
\text { AFRICA }\end{array}$ & NA & $\begin{array}{c}\text { PROBLEMS - LOW WATER STORAGE TIME (RESIDENCE TIME), HIGH } \\
\text { WATER PH (WATER QUALITY PROBLEMS), HIGH FLUORIDE } \\
\text { CONCENTRATIONS, HIGH ARSENIC CONCENTRATIONS, HIGH SULFATE } \\
\text { CONCENTRATIONS, OXYGEN PENETRATION (REDOX PROCESSES), } \\
\text { ENTRANCVE OF GAS FROM THE ATHMOSPHERE (DUE TO PHYSICAL } \\
\text { MOTIVES AND BAD DESIGN). }\end{array}$ & $\begin{array}{l}\text { (TREDOU } \\
\text { X AND } \\
\text { CAIN, } \\
2010)\end{array}$ \\
\hline $\begin{array}{l}\text { PRINCE ALBERT: } \\
\text { BOREHOLE INJECTION } \\
\text { FEASIBILITY STUDY IN } \\
\text { FRACTURED } \\
\text { SANDSTONES } \\
\end{array}$ & $\begin{array}{l}\text { DEEP } \\
\text { WELLS }\end{array}$ & $\begin{array}{l}\text { SOUTH } \\
\text { AFRICA }\end{array}$ & NA & $\begin{array}{c}\text { PROBLEMS - MICROBIOLOGICAL ISSUES, HIGH FLUORIDE } \\
\text { CONCENTRATIONS, NUTRIENTS (MAINLY NITROGEN), CLOGGING } \\
\text { (BIOLOGICAL AND CHEMICAL), IRON CONTENT, LOW QUANTITY WATER } \\
\text { AVAILABLE (CLIMATE), LOW PERMEABILITY RATES, }\end{array}$ & $\begin{array}{l}\text { (TREDOU } \\
\text { X AND } \\
\text { CAIN, } \\
2010)\end{array}$ \\
\hline
\end{tabular}




\begin{tabular}{|c|c|c|c|c|c|}
\hline CASE & $\begin{array}{l}\text { RECHAR } \\
\text { GE TYPE }\end{array}$ & $\begin{array}{l}\text { PLACE \& } \\
\text { TIME }\end{array}$ & $\begin{array}{l}\text { DURATIO } \\
\text { N OF THE } \\
\text { PROJECT }\end{array}$ & MAIN PROBLEMS & $\begin{array}{l}\text { REFEREN } \\
\text { CE }\end{array}$ \\
\hline $\begin{array}{l}\text { BITOU MUNICIPALITY } \\
\text { GROUNDWATER } \\
\text { MANAGEMENT AND } \\
\text { ARTIFICIAL RECHARGE } \\
\text { FEASIBILITY STUDY }\end{array}$ & $\begin{array}{c}\text { DEEP } \\
\text { WELLS }\end{array}$ & $\begin{array}{l}\text { SOUTH } \\
\text { AFRICA }\end{array}$ & 2 YEARS & $\begin{array}{c}\text { PROBLEMS - WATER SCARCITY (WWTP FAILURE OR TOO LOW SUPPLY } \\
\text { LIMIT), SALINITY/SODICITY, IRON CONTENT, AND ORGANIC MATTER, } \\
\text { WATER MIXTURE (CHEMICAL REACTIONS), CLOGGING (CHEMICAL AND } \\
\text { BIOLOGICAL), WATER IMBALANCE BETWEEN INJECTION AND WATER } \\
\text { INPUT (NOT ENOUGH WATER FROM REGIONAL HYDROGEOLOGY), LEGAL } \\
\text { CONSTRAINTS (OTHERS - ENVIRONMENTAL) }\end{array}$ & $\begin{array}{l}\text { (TREDOU } \\
\text { X AND } \\
\text { CAIN, } \\
2010)\end{array}$ \\
\hline $\begin{array}{l}\text { ARTIFICIAL RECHARGE } \\
\text { OF THE WINDHOEK } \\
\text { AQUIFER, NAMIBIA: } \\
\text { WATER QUALITY } \\
\text { CONSIDERATIONS }\end{array}$ & $\begin{array}{c}\text { DEEP } \\
\text { WELLS }\end{array}$ & NAMIBIA & NA & $\begin{array}{l}\text { PROBLEMS - SODICITY/SALINITY, HIGH SULFATE CONCENTRATIONS, } \\
\text { HIGH IRON CONCENTRATIONS, PRESENCE OF A DISPOSAL SITE WHICH } \\
\text { IS THE SOURCE OF ORGANIC POLLUTANTS INFILTRATION }\end{array}$ & $\begin{array}{l}\text { (TREDOU } \\
\text { X ET AL., } \\
\text { 2009) }\end{array}$ \\
\hline $\begin{array}{l}\text { IN THE FACE OF } \\
\text { CHANGING CLIMATE: } \\
\text { GROUNDWATER } \\
\text { DEVELOPMENT } \\
\text { THROUGH } \\
\text { ARTIFICIAL RECHARGE } \\
\text { IN HARD ROCK TERRAIN } \\
\text { OF KUMAUN LESSER } \\
\text { HIMALAYA }\end{array}$ & $\begin{array}{l}\text { INFILTRA } \\
\text { TION } \\
\text { BASINS }\end{array}$ & $\begin{array}{l}\text { KUMAUN } \\
\text { LESSER } \\
\text { HIMALAYA }\end{array}$ & NA & $\begin{array}{l}\text { PROBLEMS - LOW CONDUCTIVITY OF THE WATER, FLOODS, DROUGHS, } \\
\text { HIGH THICKNESS AND NOT SHALLOW AQUIFER, CIVIL WORK FAILURES } \\
\text { (OTHERS - HIGH STEEP SLOPES), WATER SCARCITY (CLIMATE, DUE TO } \\
\text { THE FACT THAT RAINFALL IS THE ONLY SOURCE OF WATER FOR THE } \\
\text { RECHARGE) }\end{array}$ & $\begin{array}{c}\text { (TRIPATHI } \\
, 2016)\end{array}$ \\
\hline $\begin{array}{l}\text { ASSESSING RISK OF } \\
\text { CLOGGING IN } \\
\text { COMMUNITY SCALE } \\
\text { MANAGED AQUIFER } \\
\text { RECHARGE SITES FOR } \\
\text { DRINKING WATER IN } \\
\text { THE } \\
\text { COASTAL PLAIN OF } \\
\text { SOUTH-WEST } \\
\text { BANGLADESH }\end{array}$ & $\begin{array}{l}\text { INFILTRA } \\
\text { TION } \\
\text { PONDS } \\
\text { AND } \\
\text { INFILTRA } \\
\text { TION } \\
\text { WELLS }\end{array}$ & $\begin{array}{c}\text { BANGLAD } \\
\text { ESH }\end{array}$ & NA & $\begin{array}{c}\text { PROBLEMS - LOW INFILTRATION RATE, CIVIL WORK FAILURE (OTHERS } \\
\text { - MAINLY RELATED TO THE MINAROLOGY OF THE TERRAIN), HIGH } \\
\text { TURBIDITY (SUSPENDED SOLIDS), HIGH SULFATES, HIGH NUTRIENTS } \\
\text { (PHOSPHORUS MAINLY), MICROBIOLOGICAL ISSUES, CLOGGING } \\
\text { (PHYSICAL TYPE MAINLY), ORGANIC MATTER CONTENT, FILTER } \\
\text { EFFICIENCY ISSUES, RESIDENCE TIME, NUTRIENTS (NITROGEN), } \\
\text { CLOGGING (BIOLOGICAL), AQUIFER HETEROGENEICITY (DIFFERENT } \\
\text { GEOLOGICAL MATERIAL LAYERS ON THE AQUIFER), WATER MIXTURE, } \\
\text { SALINITY/SODICITY ISSUES }\end{array}$ & $\begin{array}{l}\text { (SULTANA } \\
\text { AND } \\
\text { AHMED, } \\
\text { 2016) }\end{array}$ \\
\hline $\begin{array}{c}\text { INVESTIGATION OF } \\
\text { RECHARGE DYNAMICS } \\
\text { AND FLOW PATHS IN A } \\
\text { FRACTURED } \\
\text { CRYSTALLINE AQUIFER } \\
\text { IN SEMI-ARID INDIA } \\
\text { USING BOREHOLE LOGS: } \\
\text { IMPLICATIONS FOR }\end{array}$ & $\begin{array}{l}\text { PERCOLA } \\
\text { TION } \\
\text { TANK }\end{array}$ & $\begin{array}{l}\text { INDIA } \\
(\text { HYDERAB } \\
\text { AD) }\end{array}$ & NA & $\begin{array}{c}\text { PROBLEMS - GEOLOGIGAL HETEROGENEICITY (DIFFERENT GEOLOGICAL } \\
\text { MATERIAL LAYERS PRESENT), LOW INFILTRATION RATE, FLOODS, } \\
\text { DROUGHS, NOT SHALLOW AQUIFER/GEOLOGY THICKNESS, NOT } \\
\text { ENOUGH WATER (CLIMATE), WATER MIXING }\end{array}$ & $\begin{array}{l}\text { (ALAZARD } \\
\text { ET AL., } \\
\text { 2016) }\end{array}$ \\
\hline
\end{tabular}




\begin{tabular}{|c|c|c|c|c|c|}
\hline CASE & $\begin{array}{l}\text { RECHAR } \\
\text { GE TYPE }\end{array}$ & $\begin{array}{l}\text { PLACE \& } \\
\text { TIME }\end{array}$ & $\begin{array}{l}\text { DURATIO } \\
\text { N OF THE } \\
\text { PROJECT }\end{array}$ & MAIN PROBLEMS & $\begin{array}{l}\text { REFEREN } \\
\quad \text { CE }\end{array}$ \\
\hline \multicolumn{6}{|l|}{$\begin{array}{l}\text { MANAGED AQUIFER } \\
\text { RECHARGE }\end{array}$} \\
\hline $\begin{array}{l}\text { IMPACT OF A STORM- } \\
\text { WATER INFILTRATION } \\
\text { BASIN ON THE } \\
\text { RECHARGE DYNAMICS } \\
\text { IN A HIGHLY PERMEABLE } \\
\text { AQUIFER }\end{array}$ & $\begin{array}{l}\text { INFILTRA } \\
\text { TION } \\
\text { BASIN }\end{array}$ & ITALY & NA & $\begin{array}{l}\text { PROBLEMS - LEGAL CONSTRAINTS, CLOGGING (PHYSICAL AND } \\
\text { BIOLOGICAL), SUSPENDED SOLIDS, } \\
\text { NOT PROBLEM - HIGH RECHARGE RATE (PRECIPITATION), HIGH } \\
\text { AMOUNT OF WATER AVAILABLE, HIGH INFILTRATION RATE }\end{array}$ & $\begin{array}{l}\text { (MASETTI } \\
\text { ET AL., } \\
\text { 2016) }\end{array}$ \\
\hline $\begin{array}{l}\text { AN INNOVATIVE } \\
\text { ARTIFICIAL RECHARGE } \\
\text { SYSTEM TO ENHANCE } \\
\text { GROUNDWATER } \\
\text { STORAGE IN BASALTIC } \\
\text { TERRAIN: EXAMPLE } \\
\text { FROM MAHARASHTRA, } \\
\text { INDIA }\end{array}$ & $\begin{array}{l}\text { RECHAR } \\
\text { GE } \\
\text { SHAFTS } \\
\text { AND } \\
\text { SUBSURF } \\
\text { ACE } \\
\text { DAMS }\end{array}$ & INDIA & NA & $\begin{array}{c}\text { PROBLEM - LOW INFILTRATION RATE, EXCESIVE WITHDRAWAL, WATER } \\
\text { IMBALANCE (INPUT/OUTPUT OF WATER), WATER SCARCITY (CLIMATE), } \\
\text { DROUGHS, EROSION ISSUES, SUSPENDED SOLIDS }\end{array}$ & $\begin{array}{l}\text { (BHUSARI } \\
\text { ET AL., } \\
\text { 2016) }\end{array}$ \\
\hline $\begin{array}{l}\text { INTEGRATED } \\
\text { FRAMEWORKS FOR } \\
\text { ASSESSING AND } \\
\text { MANAGING HEALTH } \\
\text { RISKS IN THE CONTEXT } \\
\text { OF MANAGED AQUIFER } \\
\text { RECHARGE WITH RIVER } \\
\text { WATER }\end{array}$ & $\begin{array}{l}\text { SURFACE } \\
\text { WATER } \\
\text { FROM A } \\
\text { RIVER } \\
\text { (INFILTR } \\
\text { ATION } \\
\text { BASINS) }\end{array}$ & FINLAND & NA & $\begin{array}{c}\text { PROBLEMS - MICROBIOLOGICAL ISSUES, NUTRIENTS, CONTAMINANTS } \\
\text { (ORGANIC AND INORGANIC), LACK OF COORDINATION (POLITICAL } \\
\text { CONCERNS), ECONOMIC COSTS (DESIGN/CONSTRUCTION AND } \\
\text { OPERATION), ORGANIC MATTER, PERSISTENT ORGANIC POLUTANTS, } \\
\text { LACK OF KNOWLEDGE, COST-BENEFIT IMBALACE RELATED TO OTHER } \\
\text { WATER RESOURCES OPTIONS (WHICH WOULD BE BETTER OR } \\
\text { CHEAPER), }\end{array}$ & $\begin{array}{l}\text { (ASSMUT } \\
\text { H ET AL., } \\
\text { 2016) }\end{array}$ \\
\hline $\begin{array}{c}\text { THE EFFECTS OF } \\
\text { ARTIFiCIAL RECHARGE } \\
\text { OF GROUNDWATER ON } \\
\text { CONTROLLING LAND } \\
\text { SUBSIDENCE AND ITS } \\
\text { IFLUENCE ON } \\
\text { GROUNDWATER } \\
\text { QUALITY AND AQUIFER } \\
\text { ENERGY STORAGE IN } \\
\text { SHANGHAI, CHINA }\end{array}$ & $\begin{array}{l}\text { DEEP } \\
\text { WELLS }\end{array}$ & CHINA & NA & $\begin{array}{c}\text { PROBLEMS - SURFACE COVER OF HARD AND LOW INFILTRATION RATE } \\
\text { GEOLOGICAL MATERIALS, COMPACTION, SUBSIDENCE ISSUES, } \\
\text { GEOLOGICAL LAYERS OVERLAPPING (NOT A CONTINOUS AQUIFER BUT } \\
\text { THE DISPOSITION OF DIFFERENT GEOLOGICAL MATERIAL LAYERS), } \\
\text { AQUIFER TOO DEEP, CONTAMINANT MIGRATION, SULFATES INCREASE, } \\
\text { ORGANIC CHEMICALS INCREASE (ORGANIC CONTAMINANTS POPS), } \\
\text { NUTRIENT ISSUES (MAINLY NITROGEN), ORGANIC MATTER, CLOGGING } \\
\text { (CHEMICAL AND BIOLOGICAL) }\end{array}$ & $\begin{array}{l}\text { (SHI ET } \\
\text { AL., } \\
2016)\end{array}$ \\
\hline $\begin{array}{l}\text { IMPACT OF MANAGED } \\
\text { AQUIFER RECHARGE ON } \\
\text { THE CHEMICAL AND } \\
\text { ISOTOPIC COMPOSITION } \\
\text { OF A KARST AQUIFER, }\end{array}$ & $\begin{array}{l}\text { DEEP } \\
\text { WELLS }\end{array}$ & $\begin{array}{l}\text { JORDAN, } \\
40 \mathrm{KM} \\
\text { NEAR } \\
\text { AMMAN }\end{array}$ & NA & $\begin{array}{l}\text { PROBLEMS - LIMITED KNOWLEDGE ABOUT HYDRAULIC AND GEOLOGIC } \\
\text { CHARACTERISTICS OF THE ZONE, WATER SCARCITY (CLIMATE), } \\
\text { KARSTIC AQUIFER ISSUES (DISSOLUTION), HYDROLOGICAL } \\
\text { IMBALANCE, SALINITY ISSUES (BUT NOT DUE TO SODICITY), SULFATE }\end{array}$ & $\begin{array}{l}\text { (XANKE } \\
\text { ET AL., } \\
\text { 2015) }\end{array}$ \\
\hline
\end{tabular}




\begin{tabular}{|c|c|c|c|c|c|}
\hline CASE & $\begin{array}{l}\text { RECHAR } \\
\text { GE TYPE }\end{array}$ & $\begin{array}{l}\text { PLACE \& } \\
\text { TIME }\end{array}$ & $\begin{array}{l}\text { DURATIO } \\
\text { N OF THE } \\
\text { PROJECT }\end{array}$ & MAIN PROBLEMS & $\begin{array}{l}\text { REFEREN } \\
\quad \text { CE }\end{array}$ \\
\hline $\begin{array}{l}\text { WALA RESERVOIR, } \\
\text { JORDAN }\end{array}$ & & & & $\begin{array}{c}\text { ISSUES, NUTRIENTS, CHLORIDE, CLOGGING (PHYSICAL), SUSPENDED } \\
\text { SOLIDS, LOW INFILTRATION RATE }\end{array}$ & \\
\hline $\begin{array}{l}\text { NATURAL ATTENUATION } \\
\text { OF CHLOROBENZENE IN } \\
\text { A DEEP COFINED } \\
\text { AQUIFER DURING } \\
\text { ARTIFICIAL RECHARGE } \\
\text { PROCESS }\end{array}$ & NA & $\begin{array}{l}\text { SOUTH- } \\
\text { WEST } \\
\text { CHINA }\end{array}$ & NA & $\begin{array}{c}\text { PROBLEMS - ORGANIC CHEMICALS (POPS), SUSPENDED SOLIDS, } \\
\text { CHLORIDE, LAND USE PROBLEMS (USES OF LAND FOR AGRICULTURE, } \\
\text { INDUSTRY AND RESIDENTIAL HAVE DETERIORATED WATER QUALITY), } \\
\text { WATER USES (INDUSTRY, URBAN AND AGRICULTURE), }\end{array}$ & $\begin{array}{l}\text { (HE ET } \\
\text { AL., } \\
2016)\end{array}$ \\
\hline $\begin{array}{l}\text { ARTFICIAL RECHARGE } \\
\text { OF THE PHREATIC } \\
\text { AQUIFER IN THE UPPER } \\
\text { FRIULI PLAIN, ITALY, BY } \\
\text { A LARGE INFILTRATION } \\
\text { BASIN }\end{array}$ & $\begin{array}{l}\text { INFILTRA } \\
\text { TION } \\
\text { BASIN }\end{array}$ & ITALY & NA & $\begin{array}{c}\text { PROBLEMS - LOW PERMEABILITY, NUTRIENT ISSUES (NITROGEN } \\
\text { MAINLY), SULFATES, OVERLAPPING OF DIFFEREND GEOLOGICAL } \\
\text { LAYERS (WITH CLAY), GEOLOGICAL/HYDRAULIC INFORMATION, } \\
\text { HYDRAULIC IMBALANCE (INPUT OUTPUT OF THE RECHARGE IS } \\
\text { NEGATIVE) } \\
\text { NOT PROBLEM - LOW SALINITY }\end{array}$ & $\begin{array}{l}\text { (TEATINI } \\
\text { ET AL., } \\
\text { 2015) }\end{array}$ \\
\hline $\begin{array}{c}\text { WATER QUALITY OF THE } \\
\text { LITTLE ARKANSAS RIVER } \\
\text { AND EQUUS BEDS } \\
\text { AQUIFER BEFORE AND } \\
\text { CONCURRENT WITH } \\
\text { LARGE-SCALE } \\
\text { ARTIFICIAL RECHARGE, } \\
\text { SOUTH-CENTRAL } \\
\text { KANSAS, 1995-2012 }\end{array}$ & $\begin{array}{l}\text { DEEP } \\
\text { WELLS }\end{array}$ & $\begin{array}{l}\text { USA } \\
\text { (KANSAS) } \\
1995\end{array}$ & 6 YEARS & $\begin{array}{c}\text { PROBLEMS - CHLORIDE, NUTRIENT ISSUES (MAINLY NITROGEN), TRACE } \\
\text { ELEMENTS PROBLEMS (METALS MAINLY), NOT ENOUGH WATER } \\
\text { RECHARGED (WATER INPUT IS TOO LOW COMPARED TO THE } \\
\text { EXTRACTION AND THE TOTAL VOLUME OF THE AQUIFER), ORGANIC } \\
\text { CHEMICALS (POPS), MICROBIOLOFGICAL ISSUES (FECAL BACTERIA) }\end{array}$ & $\begin{array}{l}\text { (GALLEGO } \\
\text { S AND } \\
\text { VARELA, } \\
\text { 2015) }\end{array}$ \\
\hline $\begin{array}{l}\text { ARTIFICAL RECHARGE IN } \\
\text { LAS VEGAS VALLEY, } \\
\text { CLARK COUNTY NEVADA }\end{array}$ & $\begin{array}{l}\text { INJEECTI } \\
\text { ON } \\
\text { WELLS / } \\
\text { DEEP } \\
\text { WELLS }\end{array}$ & $\begin{array}{l}\text { USA (LAS } \\
\text { VEGAS) }\end{array}$ & NA & $\begin{array}{l}\text { PROBLEMS - HIGH THICKNESS AND NOT SHALLOW AQUIFER, SULFATE } \\
\text { CONTENT, SODIUM CONTENT, CHLORIDE CONTENT, WATER MIXTURE, } \\
\text { LOW WELL RECHARGE YIELD (PROBABLY DUE TO CLOGGING BUT } \\
\text { UNKNOWN TYPE), ECONOMIC CONSTRAINTS (OPERATONAL) }\end{array}$ & $\begin{array}{l}(\text { KATZER } \\
\text { AND } \\
\text { BROTHER } \\
\text { S, 1989) }\end{array}$ \\
\hline $\begin{array}{c}\text { WATER QUALITY } \\
\text { CHANGES RELATED TO } \\
\text { THE DEVELOPMENT OF } \\
\text { ANAEROBIC } \\
\text { CONDITIONS DURING } \\
\text { ARTIFICIAL RECHARGE } \\
\end{array}$ & $\begin{array}{l}\text { INFILTRA } \\
\text { TION } \\
\text { BASINS }\end{array}$ & $\begin{array}{l}\text { USA } \\
\text { (TEXAS) }\end{array}$ & NA & $\begin{array}{l}\text { PROBLEMS - LOW INFILTRATION RATE, SODICITY/SALINITY ISSUES, } \\
\text { HIGH SULFATE, HIGH CHLORIDE, VEGETATION/ALGAE GROWTH, } \\
\text { GENERATION OF METABOLITES (H2S), LOW PH, CLOGGING } \\
\text { (BIOLOGICAL) } \\
\text { NOT PROBLEM - LOW SUSPENDED SOLIDS CONTENT }\end{array}$ & $\begin{array}{l}\text { (WOOD } \\
\text { AND } \\
\text { BASSETT, } \\
\text { 1975) }\end{array}$ \\
\hline
\end{tabular}




\begin{tabular}{|c|c|c|c|c|c|}
\hline CASE & $\begin{array}{l}\text { RECHAR } \\
\text { GE TYPE }\end{array}$ & $\begin{array}{l}\text { PLACE \& } \\
\text { TIME }\end{array}$ & $\begin{array}{l}\text { DURATIO } \\
\text { N OF THE } \\
\text { PROJECT }\end{array}$ & MAIN PROBLEMS & $\begin{array}{l}\text { REFEREN } \\
\text { CE }\end{array}$ \\
\hline $\begin{array}{c}\text { A THIRTY YEAR } \\
\text { ARTIFICIAL RECHARGE } \\
\text { EXPERIMENT IN } \\
\text { COASTAL AQUIFER IN } \\
\text { AN ARID ZONE: THE } \\
\text { TEBOULBA AQUIFER } \\
\text { SYSTEM (TUNISIAN } \\
\text { SAHEL) }\end{array}$ & $\begin{array}{l}\text { DEEP } \\
\text { WELLS / } \\
\text { INJECTIO } \\
\text { N WELLS }\end{array}$ & $\begin{array}{l}\text { TUNISIA } \\
(\text { SAHEL) } \\
1972- \\
2002\end{array}$ & 30 YEARS & $\begin{array}{c}\text { PROBLEMS - WATER SCARCITY (DUE TO CLIMA AND PRECIPITATION), } \\
\text { LOW QUANTITY OF WATER RESOURCES AVAILABLE FOR RECHARGE, } \\
\text { HIGH SALINITY/SODICITY, LOW INFILTRATION RATE, LOW POROSITY, } \\
\text { REGIONAL HYDROGEOLOGY PROBLEMS (NEGATIVE INPUT/OUTPUT } \\
\text { RATIO) } \\
\text { NOT PROBLEM - CHEAPER WATER PRICES, COMPARED TO OTHER } \\
\text { TECHNOLOGIES THE PRICES AND COSTS (DESIGN/CONSTRUCTION AND } \\
\text { OPERATION) ARE BETTER IN AQUIFER ARTIFICIAL RECHARGE }\end{array}$ & $\begin{array}{l}\text { (BOURI } \\
\text { AND } \\
\text { DHIA } \\
2010)\end{array}$ \\
\hline $\begin{array}{c}\text { ESTIMATING } \\
\text { GROUNDWATER } \\
\text { RECHARGE INDUCED BY } \\
\text { ENGINEERING } \\
\text { SYSTEMS IN A SEMIARID } \\
\text { AREA (SOUTHEASTERN } \\
\text { SPAIN) }\end{array}$ & $\begin{array}{l}\text { INFILTRA } \\
\text { TION } \\
\text { BASINS } \\
\text { (VIA } \\
\text { CHECK } \\
\text { DAMS } \\
\text { AND } \\
\text { GRAVEL } \\
\text { PITS) }\end{array}$ & $\begin{array}{c}\text { SPAIN } \\
\text { (ALMERIA } \\
\text { ) }\end{array}$ & NA & $\begin{array}{c}\text { PROBLEMS - HIGH SLOPE, WATER SCARCITY (CLIMATE), CLOGGING } \\
\text { (PHYSICAL) } \\
\text { NOT PROBLEM - GOOD INFILTRATION RATE }\end{array}$ & $\begin{array}{l}\text { (MARTÍN- } \\
\text { ROSALES } \\
\text { ET AL.' } \\
\text { 2007) }\end{array}$ \\
\hline $\begin{array}{c}\text { QUANTITATIVE PCR } \\
\text { MONITORING OF } \\
\text { ANTIBIOTIC } \\
\text { RESISTANCE GENES AND } \\
\text { BACTERIAL PATHOGENS } \\
\text { IN THREE EUROPEAN } \\
\text { ARTIFICIAL } \\
\text { GROUNDWATER } \\
\text { RECHARGE SYSTEMS }\end{array}$ & $\begin{array}{l}\text { RIVER } \\
\text { INFILTRA } \\
\text { TION }\end{array}$ & $\begin{array}{c}\text { SPAIN } \\
\text { (SABADEL } \\
\text { L) }\end{array}$ & 1 YEAR & $\begin{array}{c}\text { PROBLEMS - MICROBIOLOGICAL ISSUES, LEGAL CONSTRAINTS } \\
\text { (DOESN'T COMPLY WITH DRINKING STANDARDS) }\end{array}$ & $\begin{array}{l}\text { (BÖCKEL } \\
\text { MANN ET } \\
\text { AL.' } \\
\text { 2009) }\end{array}$ \\
\hline $\begin{array}{l}\text { QUANTITATIVE PCR } \\
\text { MONITORING OF } \\
\text { ANTIBIOTIC } \\
\text { RESISTANCE GENES AND } \\
\text { BACTERIAL PATHOGENS } \\
\text { IN THREE EUROPEAN } \\
\text { ARTIFICIAL } \\
\text { GROUNDWATER } \\
\text { RECHARGE SYSTEMS }\end{array}$ & $\begin{array}{l}\text { DEEP } \\
\text { WELLS }\end{array}$ & $\begin{array}{l}\text { ITALY } \\
\text { (NARDÒ) }\end{array}$ & 1 YEAR & $\begin{array}{c}\text { PROBLEMS - LOW PH (POSSIBLY METAL DISSOLUTION AND } \\
\text { MOBILISATION), WATER MIXTURE, MICROBIOLOGICAL ISSUES, WATER } \\
\text { SCARCITY (CLIMATE), LEGAL CONSTRAINTS (DOESN'T COMPLY WITH } \\
\text { DRINKING STANDARDS) }\end{array}$ & $\begin{array}{l}\text { (BÖCKEL } \\
\text { MANN ET } \\
\text { AL.' } \\
\text { 2009) }\end{array}$ \\
\hline
\end{tabular}




\begin{tabular}{|c|c|c|c|c|c|}
\hline CASE & $\begin{array}{l}\text { RECHAR } \\
\text { GE TYPE }\end{array}$ & $\begin{array}{l}\text { PLACE \& } \\
\text { TIME }\end{array}$ & $\begin{array}{l}\text { DURATIO } \\
\text { N OF THE } \\
\text { PROJECT }\end{array}$ & MAIN PROBLEMS & $\begin{array}{l}\text { REFEREN } \\
\text { CE }\end{array}$ \\
\hline $\begin{array}{c}\text { QUANTITATIVE PCR } \\
\text { MONITORING OF } \\
\text { ANTIBIOTIC } \\
\text { RESISTANCE GENES AND } \\
\text { BACTERIAL PATHOGENS } \\
\text { IN THREE EUROPEAN } \\
\text { ARTIFICIAL } \\
\text { GROUNDWATER } \\
\text { RECHARGE SYSTEMS }\end{array}$ & $\begin{array}{l}\text { INFILTTR } \\
\text { ATION } \\
\text { BASINS }\end{array}$ & $\begin{array}{l}\text { BELGIUM } \\
\text { /TORREEL } \\
\text { E) }\end{array}$ & 1 YEAR & $\begin{array}{c}\text { PROBLEMS - WATER MIXTURE, MICROBIOLOGICAL ISSUES, DESIGN } \\
\text { AND CONSTRUCTION COSTS, OPERATIONAL COSTS (REVERSE OSMISIS } \\
\text { AND ULTRAFILTRATION TREATMENTS), }\end{array}$ & $\begin{array}{l}\text { (BÖCKEL } \\
\text { MANN ET } \\
\text { AL., } \\
\text { 2009) }\end{array}$ \\
\hline $\begin{array}{c}\text { MODELING SEASONAL } \\
\text { REDOX DYNAMICS } \\
\text { AND THE } \\
\text { CORRESPONDING FATE } \\
\text { OF THE } \\
\text { PHARMACEUTICAL } \\
\text { RESIDUE PHENAZONE } \\
\text { DURING ARTIFICIAL } \\
\text { RECHARGE OF } \\
\text { GROUNDWATER } \\
\end{array}$ & $\begin{array}{l}\text { DEEP } \\
\text { WELLS }\end{array}$ & $\begin{array}{l}\text { GERMANY } \\
\text { (BERLIN) }\end{array}$ & NA & $\begin{array}{c}\text { PROBLEMS - CLOGGING (UNKNOWN TYPE), LOW INFILTRATION RATE } \\
\text { (PERIODICALLY CHANGING THIS RATE DUE TO CLOGGING ISSUES), } \\
\text { NUTRIENTS, LOW NATURAL ATENUATION }\end{array}$ & $\begin{array}{l}\text { (GRESKO } \\
\text { WIAK ET } \\
\text { AL., } \\
\text { 2006) }\end{array}$ \\
\hline $\begin{array}{l}\text { INTEGRATED WATER } \\
\text { MANAGEMENT FOR THE } \\
\text { 21ST CENTURY: } \\
\text { PROBLEMS AND } \\
\text { SOLUTIONS }\end{array}$ & NA & NA & NA & $\begin{array}{l}\text { PROBLEMS - LEGAL (OWNERSHIP,REUSE), CONTAMINATION (EXTERNAL } \\
\text { POLUTANTS AND WASTE WATER RECHARGED POLLUTANTS), SALINITY, } \\
\text { WATER SHORTAGE DUE TO CLIMATE CHANGE, FLOODING DUE TO } \\
\text { CLIMATE CHANGE, NOT ENOUGH WATER EXTRACTED FROM MAR. }\end{array}$ & $\begin{array}{l}\text { (BOUWER } \\
, \text { 2002B) }\end{array}$ \\
\hline $\begin{array}{c}\text { GROUNDWATER } \\
\text { RECHARGE WITH } \\
\text { RECLAIMED MUNICIPAL } \\
\text { WASTEWATER: HEALTH } \\
\text { AND REGULATORY } \\
\text { CONSIDERATIONS } \\
\text { TAKASHI }\end{array}$ & $\begin{array}{l}\text { SURFACE } \\
\text { SPREADI } \\
\text { NG AND } \\
\text { INJECTIO } \\
\text { N WELLS }\end{array}$ & NA & NA & $\begin{array}{l}\text { PROBLEMS - LEGAL (HEALTH LAWS), CONTAMINATION (EXTERNAL, } \\
\text { TRACE CONTAMINANTS, ETC.), LACK OF KNOWLEDGE ABOUT THE RISKS } \\
\text { AND PROBLEMS, }\end{array}$ & $\begin{array}{l}\text { (ASANO } \\
\text { AND } \\
\text { COTRUVO } \\
\text {, 2004) }\end{array}$ \\
\hline $\begin{array}{l}\text { FUTURE MANAGEMENT } \\
\text { OF AQUIFER RECHARGE }\end{array}$ & ALL & NA & NA & $\begin{array}{c}\text { PROBLEMS - WATERLOGGING, DAMAGE TO STRUCTURES, FLOODING, } \\
\text { SOIL SALINISATION, LEGAL ISSUES, WATER QUALITY, LACK OF } \\
\text { KNOWLEDGE }\end{array}$ & $\begin{array}{l}\text { (DILLON, } \\
2005)\end{array}$ \\
\hline
\end{tabular}




\begin{tabular}{|c|c|c|c|c|c|}
\hline CASE & $\begin{array}{l}\text { RECHAR } \\
\text { GE TYPE }\end{array}$ & $\begin{array}{l}\text { PLACE \& } \\
\text { TIME }\end{array}$ & $\begin{array}{l}\text { DURATIO } \\
\text { N OF THE } \\
\text { PROJECT }\end{array}$ & MAIN PROBLEMS & $\begin{array}{l}\text { REFEREN } \\
\text { CE }\end{array}$ \\
\hline $\begin{array}{l}\text { MANAGED AQUIFER } \\
\text { RECHARGE: AN } \\
\text { ASSESSMENT OF ITS } \\
\text { ROLE AND } \\
\text { EFFECTIVENESS IN } \\
\text { WATERSHED } \\
\text { MANAGEMENT } \\
\end{array}$ & $\begin{array}{l}\text { INFILTRA } \\
\text { TION } \\
\text { BASIN } \\
\text { AND } \\
\text { CHECK } \\
\text { DAMS }\end{array}$ & $\begin{array}{l}\text { KOLWAN } \\
\text { VALLEY } \\
1998\end{array}$ & NA & $\begin{array}{l}\text { PROBLEMS - WATER SCARCITY (TO INFILTRATE), LACK OF } \\
\text { INFORMATION, LACK OF WORKFORCE, MAINTENANCE COSTS, LEGAL } \\
\text { ISSUES, OWNERSHIP ISSUES, COST-BENEFIT SHRING ISSUES, } \\
\text { TENSIONS BETWEEN GOVERNMENT/AGENCIES/PEOPLE, INCREASED } \\
\text { WATER ABSTRACTION, CLIMATE CHANGE ISSUES, CLOGGING. }\end{array}$ & $\begin{array}{l}\text { (GALE ET } \\
\text { AL., } \\
\text { 2006) }\end{array}$ \\
\hline $\begin{array}{c}\text { MANAGED AQUIFER } \\
\text { RECHARGE: AN } \\
\text { ASSESSMENT OF ITS } \\
\text { ROLE AND } \\
\text { EFFECTIVENESS IN } \\
\text { WATERSHED } \\
\text { MANAGEMENT }\end{array}$ & $\begin{array}{c}\text { CHECK } \\
\text { DAM } \\
\text { AND } \\
\text { PERCOLA } \\
\text { TION } \\
\text { TANK }\end{array}$ & $\begin{array}{c}\text { SATLASA } \\
\text { NA } \\
\text { (MUMANV } \\
\text { AS, } \\
\text { BHANAVA } \\
\text { S 1, } \\
\text { BBHANAV } \\
\text { AS } 2 \text { AND } \\
\text { SAMRAPU } \\
\text { R) } 2001- \\
2003\end{array}$ & NA & $\begin{array}{c}\text { PROBLEMS - INVESTEMENT COSTS ISSUES, LAND USE (19,5 KM2), } \\
\text { CLOGGING, LOW WATER RETENTION, HIGH FLUORIDE, HIGH NA, HIGH } \\
\text { NO3-, }\end{array}$ & $\begin{array}{l}\text { (GALE ET } \\
\text { AL., } \\
\text { 2006) }\end{array}$ \\
\hline $\begin{array}{l}\text { MANAGED AQUIFER } \\
\text { RECHARGE: AN } \\
\text { ASSESSMENT OF ITS } \\
\text { ROLE AND } \\
\text { EFFECTIVENESS IN } \\
\text { WATERSHED } \\
\text { MANAGEMENT }\end{array}$ & $\begin{array}{c}\text { INFILTRA } \\
\text { TION } \\
\text { BASIN } \\
\text { AND } \\
\text { CHECK } \\
\text { DAM }\end{array}$ & $\begin{array}{l}\text { COIMBAT } \\
\text { ORE } \\
\text { (KARNAM } \\
\text { PETTAI, } \\
\text { KODAGIP } \\
\text { ALAYA1 } \\
\text { AND } \\
\text { KODANGI } \\
\text { PALAYAM } \\
\text { 2) }\end{array}$ & NA & $\begin{array}{c}\text { PROBLEMS - LAND USE, SOCIAL ACCEPTANCE, HIGH COSTS, LEGAL } \\
\text { ISSUES, OWNERSHIP ISSUES, LACK OF MAINTENANCE (DUE TO COSTS, } \\
\text { LACK OF KNOWLEDGE AND UNWILLINGNESS), LOW INFILTRATION } \\
\text { RATES (MAINLY DUE TO CLOGGING), HIGH CONCENTRATIONS OF BA, } \\
\text { FE AND NO3- }\end{array}$ & $\begin{array}{l}\text { (GALE ET } \\
\text { AL., } \\
\text { 2006) }\end{array}$ \\
\hline $\begin{array}{c}\text { ORGANIC } \\
\text { MICROPOLLUTANT } \\
\text { REMOVAL FROM } \\
\text { WASTEWATER } \\
\text { EFFLUENT-IMPACTED } \\
\text { DRINKING WATER } \\
\text { SOURCES DURING BANK } \\
\text { FILTRATION AND } \\
\text { ARTIFICIAL RECHARGE }\end{array}$ & $\begin{array}{c}\text { BANK } \\
\text { FILTRATI } \\
\text { ON AND } \\
\text { INFILTRA } \\
\text { TION } \\
\text { BASIN }\end{array}$ & $\begin{array}{c}\text { GERMANY } \\
2002\end{array}$ & 3 YEARS & $\begin{array}{c}\text { PROBLEMS - VERY HIGH RETENTION TIME FOR BANK FILTRATION, VERY } \\
\text { SHORT RETENTION TIME FOR INFILTRATION BASIN, BOTH HAD } \\
\text { PROBLEMS WITH WATER QUALITY (MICROPOLLUTANTS, MOSTLY } \\
\text { PHARMACEUTICAL PRODUCTS). }\end{array}$ & $\begin{array}{l}\text { (MAENG } \\
\text { ET AL., } \\
\text { 2010) }\end{array}$ \\
\hline
\end{tabular}




\begin{tabular}{|c|c|c|c|c|c|}
\hline CASE & $\begin{array}{l}\text { RECHAR } \\
\text { GE TYPE }\end{array}$ & $\begin{array}{l}\text { PLACE \& } \\
\text { TIME }\end{array}$ & $\begin{array}{l}\text { DURATIO } \\
\text { N OF THE } \\
\text { PROJECT }\end{array}$ & MAIN PROBLEMS & $\begin{array}{l}\text { REFEREN } \\
\text { CE }\end{array}$ \\
\hline $\begin{array}{l}\text { BIOGEOCHEMICAL } \\
\text { PROCESSES DURING } \\
\text { THE INFILTRATION OF } \\
\text { RIVER WATER INTO AN } \\
\text { ALLUVIAL AQUIFER }\end{array}$ & $\begin{array}{l}\text { RIVERBA } \\
\text { NK } \\
\text { FILTRATI } \\
\text { ON }\end{array}$ & FRANCE & NA & $\begin{array}{c}\text { PROBLEMS - SEDIMENTS WHERE WATER INFILTRATES ARE } \\
\text { CONTAMINATED WITH ZINC AND CADMIUM }\end{array}$ & $\begin{array}{r}(\mathrm{SITE} \\
19 \\
93)\end{array}$ \\
\hline $\begin{array}{c}\text { USE OF STATIC } \\
\text { QUANTITATIVE } \\
\text { MICROBIAL RISK } \\
\text { ASSESSMENT TO } \\
\text { DETERMINE PATHOGEN } \\
\text { RISKS IN AN } \\
\text { UNCONFINED } \\
\text { CARBONATE AQUIFER } \\
\text { USED FOR MANAGED } \\
\text { AQUIFER RECHARGE } \\
\text { SIMON }\end{array}$ & $\begin{array}{l}\text { INJECTIO } \\
\text { N WELLS }\end{array}$ & $\begin{array}{l}\text { AUSTRALI } \\
\text { A }\end{array}$ & NA & PROBLEMS - CHEMICAL CLOGGING & $\begin{array}{l}\text { (TOZE ET } \\
\text { AL., 2010) }\end{array}$ \\
\hline $\begin{array}{c}\text { STUDY OF THE } \\
\text { FEASIBILITY OF AN } \\
\text { AQUIFER STORAGE AND } \\
\text { RECOVERY SYSTEM IN A } \\
\text { DEEP AQUIFER IN } \\
\text { BELGIUM }\end{array}$ & $\begin{array}{l}\text { INJECTIO } \\
\text { N WELLS }\end{array}$ & BELGIUM & NA & $\begin{array}{l}\text { PROBLEMS - QUALITY ISSUES DUE TO WATER MIXING, LOW AMOUNT } \\
\text { OF RECOVERED WATER, QUALITY ISSUES (PHOSPHATE, WATER } \\
\text { COLOUR, DOC), CLOGGING (PHYSICAL AND BIOLOGICAL). }\end{array}$ & $\begin{array}{l}\text { (VANDENB } \\
\text { OHEDE ET } \\
\text { AL., 2008) }\end{array}$ \\
\hline $\begin{array}{c}\text { EFFECTIVENESS OF } \\
\text { RIVERBANK FILTRATION } \\
\text { FOR REMOVAL OF } \\
\text { NITROGEN FROM } \\
\text { HEAVILY POLLUTED } \\
\text { RIVERS: A CASE STUDY } \\
\text { OF KUIHE RIVER, } \\
\text { XUZHOU, JIANGSU, } \\
\text { CHINA }\end{array}$ & $\begin{array}{c}\text { RIVERBA } \\
\text { NK } \\
\text { FILTRATI } \\
\text { ON }\end{array}$ & CHINA & NA & $\begin{array}{c}\text { PROBLEMS - QUALITY ISSUES (HIGH NITROGEN CONCENTRATIONS), } \\
\text { LOW DEGRADATION OF POLLUTANTS }\end{array}$ & $\begin{array}{l}(\text { WU ET } \\
\text { AL., 2007) }\end{array}$ \\
\hline
\end{tabular}

* MONITORING, DATA COLLECTION AND ZONE STUDY ARE ASPECTS THAT SHOULD BE TAKEN INTO ACCOUNT BECAUSE THEY CAN LEAD TO FAILURE OF THE MAR FACILITY IF THEY ARE NOT DONE CORRECTLY (MISSINFORMATION OF THE MEASURES, UNACCEPTABLE COSTS OR BAD SPATIAL DISTRIBUTION)

*CORROSION OF METAL COMPONENTS IN THE PIPES AND MACHINES IS A COMMON PROBLEM. 
*INSUFICIENT EVIDENCE FOR RISK QUANTIFICATION FOR SOME ISSUES (LIKE INORGANIC CHEMICALS)

*THERE'S USUALLY THE NEED OF WWTP AND DESSALATION PLANTS TO TREAT THE WATER PRIOR TO INJECTION (MOST CASES)

*USUALLY THERE'S A LACK OF REGULATORY PRECEDENT

*USUALLY ARTIFICIAL RECHARGE IMPLIES WATER PUMPING (FOR AGRICULTURAL, INDUSTRIAL OR URBAN USES) SO MAYBE THERE'S AN IMBALANCE BETWEEN THE WATER ENTERING THE SYSTEM AND THE WATER BEING PUMPED OUT OF IT

*THERE SHOULD BE INCLUDED A FAILURE DUE TO LATERAL INFILTRATION INSTEAD OF VERTICAL INFILTRATION

*GENERATION OF METABOLITES IS AN ISSUE THAT USUALLY HAPPENS WHERE THERE ARE MICROBIOLOGICAL PROBLEMS

*STORM QUALITY MONITORING IS IMPORTANT IN ORDER TO IDENTIFY AND CONTROL WATER INPUTS TO THE SYSTEM (KNOWING THEIR QUALITY AND QUANTITY)

*SOMETIMES THE PROBLEM IS NOT THE INFILTRATION RATE BUT THE POROSITY OR CONDUCTIVITY 


\section{S2. LITERARURE REVIEW OF MANAGED AQUIFER RECHARGE FAILURES}

$\begin{array}{ll}\text { LEG } & \text { Legal constraints - Changes in the legislation or failures to comply } \\ & \text { with its requirements (especially for health or environmental } \\ \text { TER } & \text { Tegislation). } \\ \text { EU } & \text { European } \\ \text { NAT } & \text { National } \\ \text { REG } & \text { Regional/Local } \\ \text { SC } & \text { Scope of legislation - Depending on the legislation type, its } \\ & \text { restrictions and risk probabilities can be significantly different. } \\ \text { HTH } & \text { Health legislation } \\ \text { OTH } & \text { Others - Mainly environmentally related legislation that can pose } \\ & \text { Some kind of restrictions to the operation of the Mar facility. } \\ \text { ECO } & \text { Economic constraints } \\ \text { MAC } & \text { Macroeconomic constraints - Restrictions related to global effects } \\ & \text { on economy like crisis, changes in currency value, increase of the } \\ & \text { petroleum price, etc. That may cause a reduction on the interest } \\ \text { in recharging water or a reduction on the quantity recharged. } \\ \text { MIC } & \text { Microeconomic constraints } \\ \text { NEWR } & \text { Not enough water to recharge due to other economical uses } \\ \text { IND } & \text { Industrial use } \\ \text { AGR } & \text { Agricultural use } \\ \text { DOM } & \text { Domestic use } \\ \text { COST } & \text { Cost restriction } \\ \text { LWP } & \text { Low price of water - MAR has fixed costs and the water that }\end{array}$ produces has a fixed price, however other water sources can have a variable price that may be cheaper than the water obtained by MAR sources and therefore, the incentive for developing MAR is lowered.

HCST High installation cost

MAIN

T

High maintenance cost/maintenance requirements

FUND Lack of private/public funding

So Social unacceptance - Neighbors and other citizens may dislike MAR due to their own opinions according to safety, health, noise, etc.

HTHR Health risk perception HCOS

$\mathbf{T}$

High cost perception

BRH Behavioral requirements - MAR as any technical facility has safety procedures and a code of conduct for the workers and people related to the facility operation. Thus implying that people may be unwilling to accept a MAR facility because they fear a facility that needs safety requirements like these.

CHILD Children surveillance

FAIR Fair distribution of treated water 


$\begin{array}{ll}\text { EFECT } & \text { Perception of effectiveness } \\ \text { GOV } & \text { Governance } \\ \text { CORD } & \text { Lack of coordination } \\ \text { NTK } & \text { Non-technical knowledge } \\ \text { SD } & \text { Structural Damages } \\ \text { FL } & \text { Flooding } \\ \text { NH } & \text { Natural hazards (e.g. earthquake) } \\ \text { TA } & \text { Terrorism activities/Vandalism } \\ \text { CV } & \text { Civil work failures }\end{array}$

SLP Slope stability - Mostly for surface infiltration, it implies that the water recharge or accumulation in storage ponds may cause instabilities in the slopes and possibly causing landfalls or wall breakages.

PB Pipe breakage

OTH Others

AD Aquifer dissolution (e.g. in karstic aquifer)

QUAT Not enough water recharged

LWIP Low quality water

BIO Sanitary/biological restrictions (e.g. due the pathogens)

PHY Physical restrictions

TP Turbidity/particles

CHE Chemical restrictions

MET Metals (e.g. arsenic, manganese)

SL Salinity and sodicity

NUT Nutrients (nitrogen, phosphorous)

OC Organic chemicals (pollutants, EOCs)

RN Radionuclides

WS Water scarcity

CLIM Climate

DRO Droughts

WWTP Waste water treatment plant failure

LAND Desalination plant failure

RIV River regulation

CLOG Clogging 
PCL Physical clogging

FDP Failure deposition pond (particles from diverted water) - The particles may not be sedimented efficiently and still be present in the recharged water, diminishing and difficulting the entrance of water during recharge operations.

PPF Pipe filter fails

RT Residence time - Not enough residence time may cause an inefficient reduction and degradation of contaminants and pathogens.

SFP Source fine particles (generation inside MAR facility)

TD Transport sedimentation (erosion or deposition from recharge pond)

DP Deposition

ER Erosion

BCL Bioclogging

CCL Chemical clogging

EV Evaporation (excess)

WMX Water mixtures - The combination of the aquifer natural water and the injected water may cause precipitation of minerals and therefore chemical clogging, reducing the amount of water recharged into the aquifer.

MIC Microbial population catalysis - Microbial population may cause changes in the water chemistry which in turn can imply precipitation of minerals or other byproducts (microbial biofilms) which can reduce recharged water.

com Compaction

GAS Generation of gas (e.g. bubble formation)

PHM Physical Motives

BAC Bacterial processes

ID Inappropriate design

QUAL Unacceptable quality of water at sensitive location

AN Inefficient natural attenuation

OM Organic matter

EOC Emerging organic compounds

UN Nutrients

MET Generation of metabolites - The chemicals injected or already present in the aquifer may be degraded and then transformed into another compounds which may be equally or more dangerous 
than the previous ones, therefore affecting the quality of the water.

NC Nitrogen cycle $\left(\mathrm{NO}_{2}^{-}, \mathrm{N}_{2} \mathrm{O} \ldots\right)$

EOC Emerging organic compounds

$\mathbf{H}_{2} \mathbf{S}$ Other nutrient cycles $\left(\mathrm{H}_{2} \mathrm{~S}\right)$

MOB Mobilization

MET Metals

ST Specific targets - A MAR project can be done for many reasons but there is always one or a couple of them that are the main ones for the project. Those reasons may have specific objectives that need to be achieved in order to have a successful MAR project.

SWB Seawater barriers

PROT Protected water body

WL Water levels

RIV River

SPR Spring

WET Wetland 


\section{S3. RISKMAR APP MANUAL}

The programming language of the MAR-RISKAPP was Visual Basic. Which is based on the usage of macros, which are usually short programing code lines that are used to give some kind of orders to the program in order to do some specific calculations or to set automatically some kind of properties for the working environment (among other kind of possibilities) and objects.

The result was the MAR-RISKAPP tool which allows the user to define four probability categories (high risk, medium risk, low risk and no risk) of failure for each type MAR event and therefore calculate the risk of a general MAR failure (representing the different probabilities within a fault tree).

The MAR-RISKAPP was structured in four main steps: 1) HOME, 2) INPUT, 3) RESULTS and 4) GRAPHICAL RESULTS.

The application starts with the HOME step. This first stage shows the name of the tool, the creators and the main institutions involved in it, with a clear indication that the tool was developed within the framework of project MARSOL. From this starting point, there are two possible ways to proceed: 1) HELP (which sends the user to a general explanation of the tool and its operational set can be found), and 2) START (which sends the user to the second step of the tool - INPUT). 


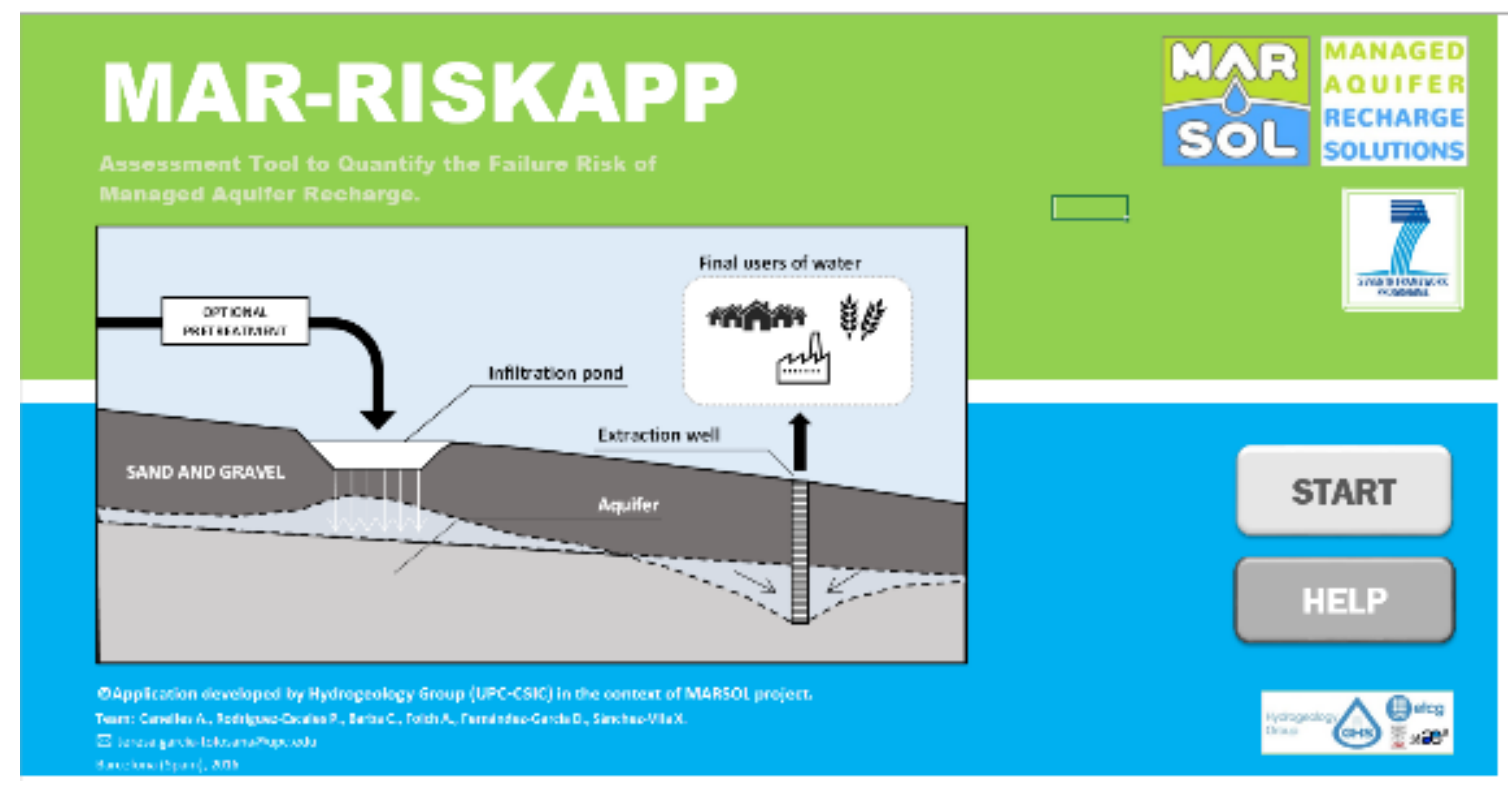

Figure S3.1. Home layout visualization.

The second step of the tool is INPUT. At this point, the user has to choose the risk category of the different events for both non-technical and technical issues (the Input sheet can be seen in Figure S3.2). This step implies that data has to be filled in four different sheets: 1) NON-TECHNICAL CONSTRAINTS - DESIGN AND CONSTRUCTION (Figure S3.3), 2) TECHNICAL CONSTRAINTS - DESIGN AND CONSTRUCTION (Figure S3.4), 3) NONTECHNICAL CONSTRAINTS - OPERATION (Figure S3.5) and 4) TECHNICAL CONSTRAINTS - OPERATION (Figure S3.6). Where the user has to answer for all the events by writing an " $X$ " on the risk category that they think it is the most adequate.

Only one " $X$ " has to be written at each line, as the person filling the sheet must select one of the following four categories of risk: no risk, high risk, medium risk, or low risk. In the Input worksheet, there is a button of instructions; when this button is clicked, a pop-up text box is shown (which indicates the order that the four input sheets should be filled and some explanation about their meaning). In addition, each input sheet has its own instruction button, which explains the user by using text and images, how to fill the surveys from each input worksheet. Finally, when all the input sheets have been filled, the user can run the Results button in order to go the RESULTS sheet (or if the user need help, the Help button can bring him/her 
to the Help sheet, or if the user wants to go back to the HOME sheet, he/she can press the BACK TO HOME button).

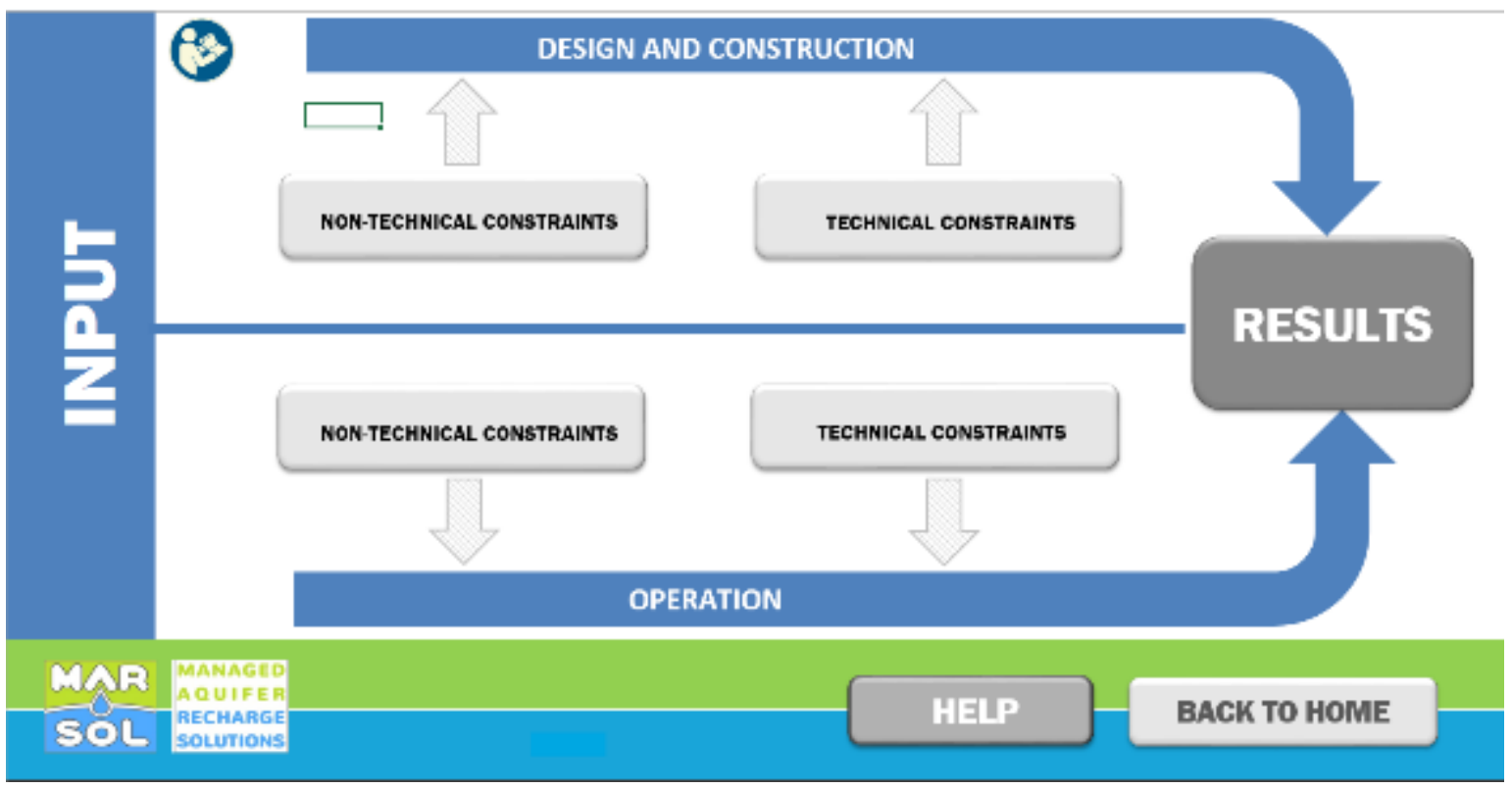

Figure S3.2. Input layout visualization

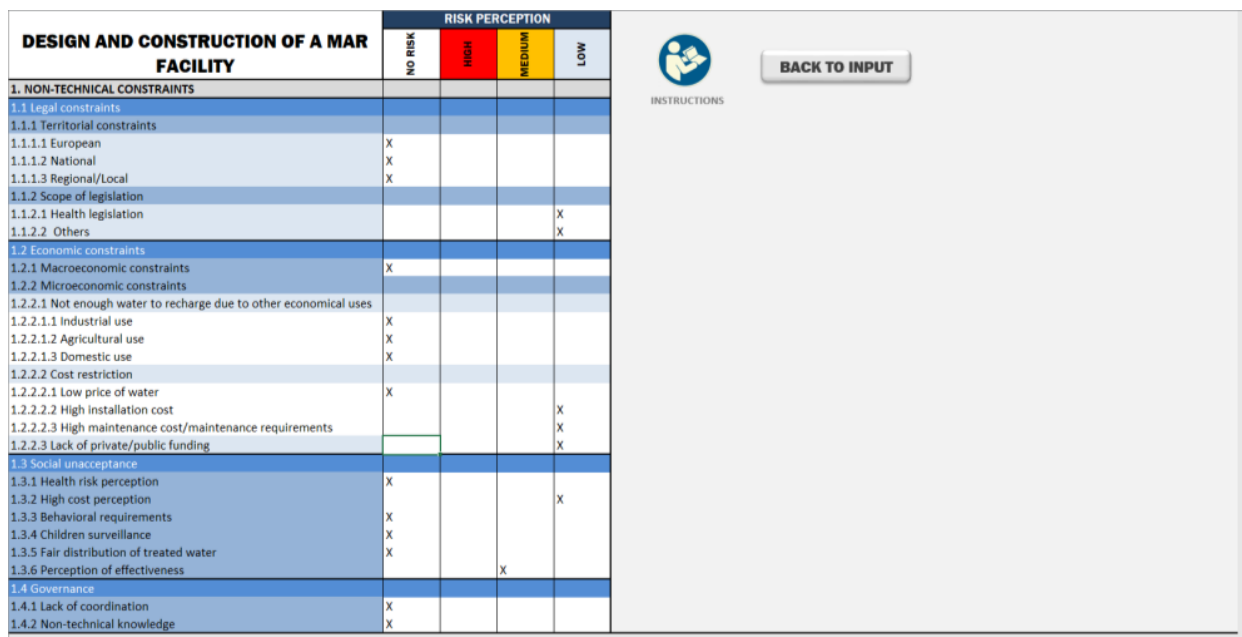

Figure S3.3. Non-technical constraints - Design and construction, sheet visualization. 


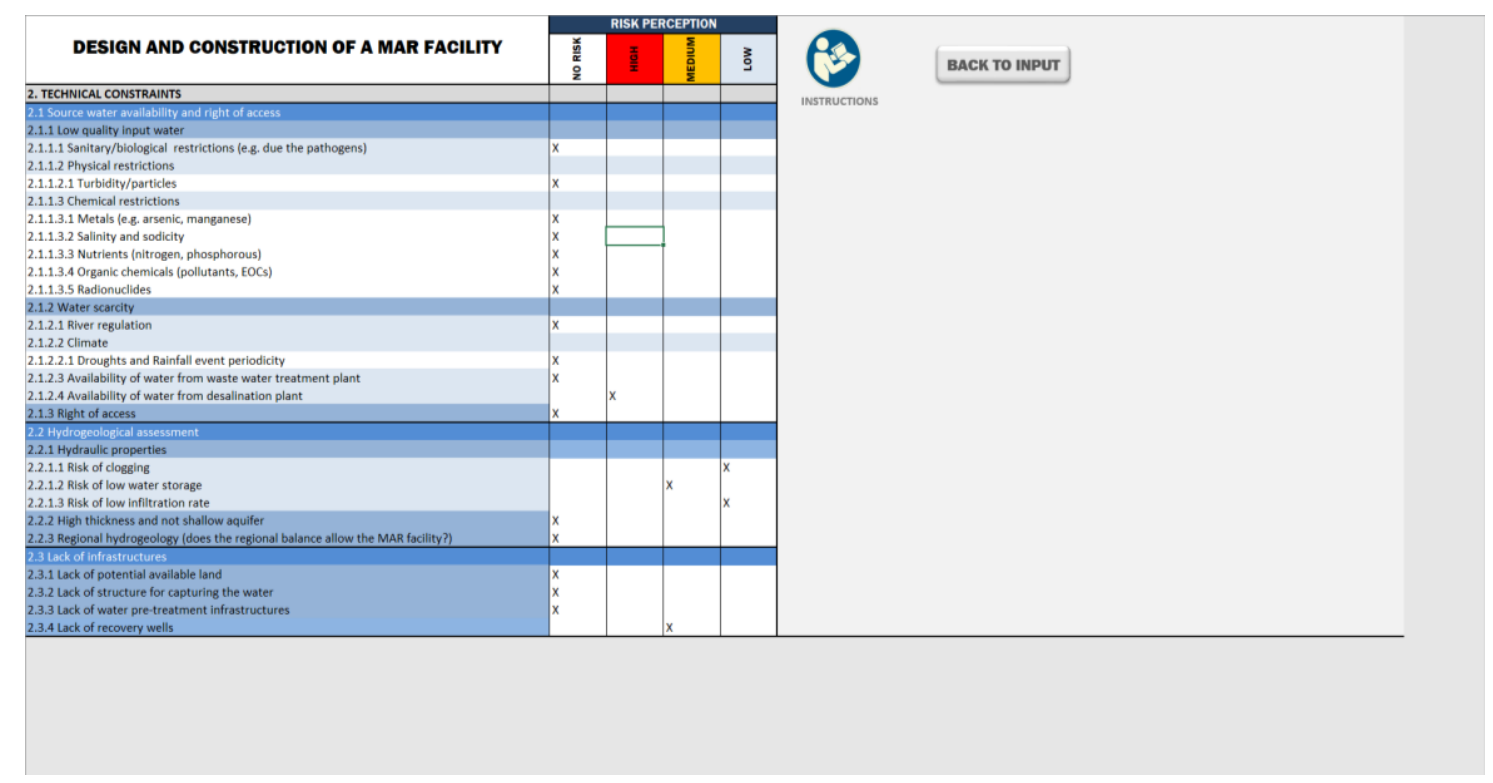

Figure S3.4. Technical constraints - Design and construction, sheet visualization.

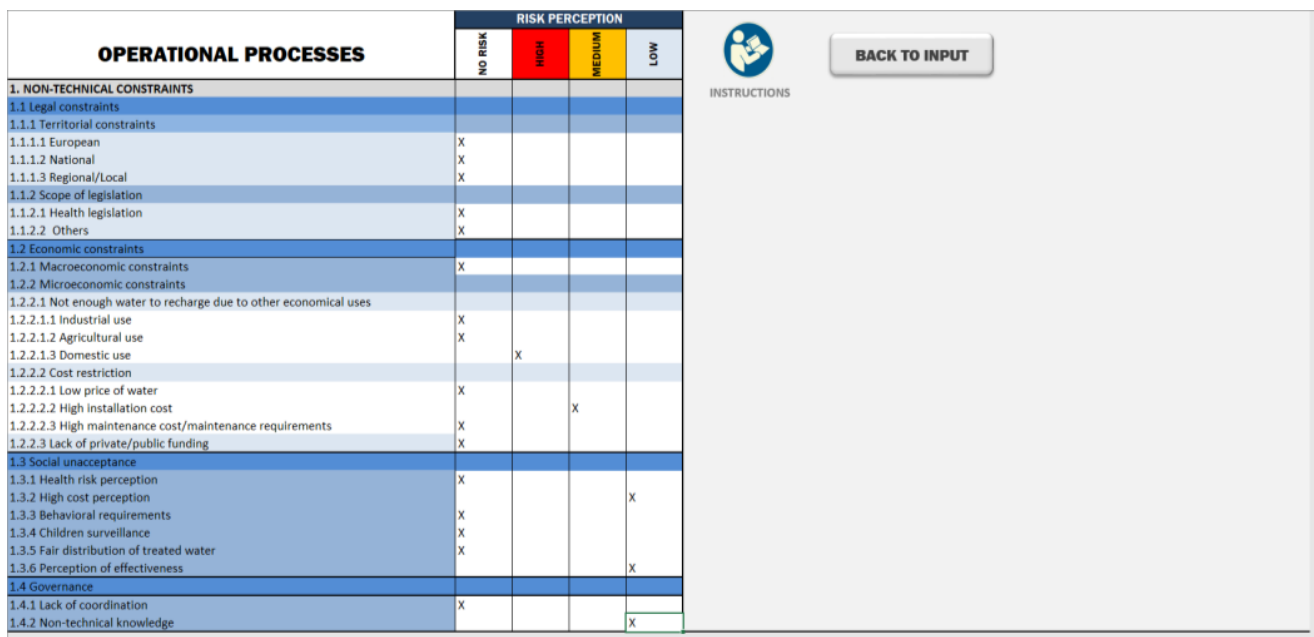

Figure S3.5. Non-technical constraints - Operation, sheet visualization. 


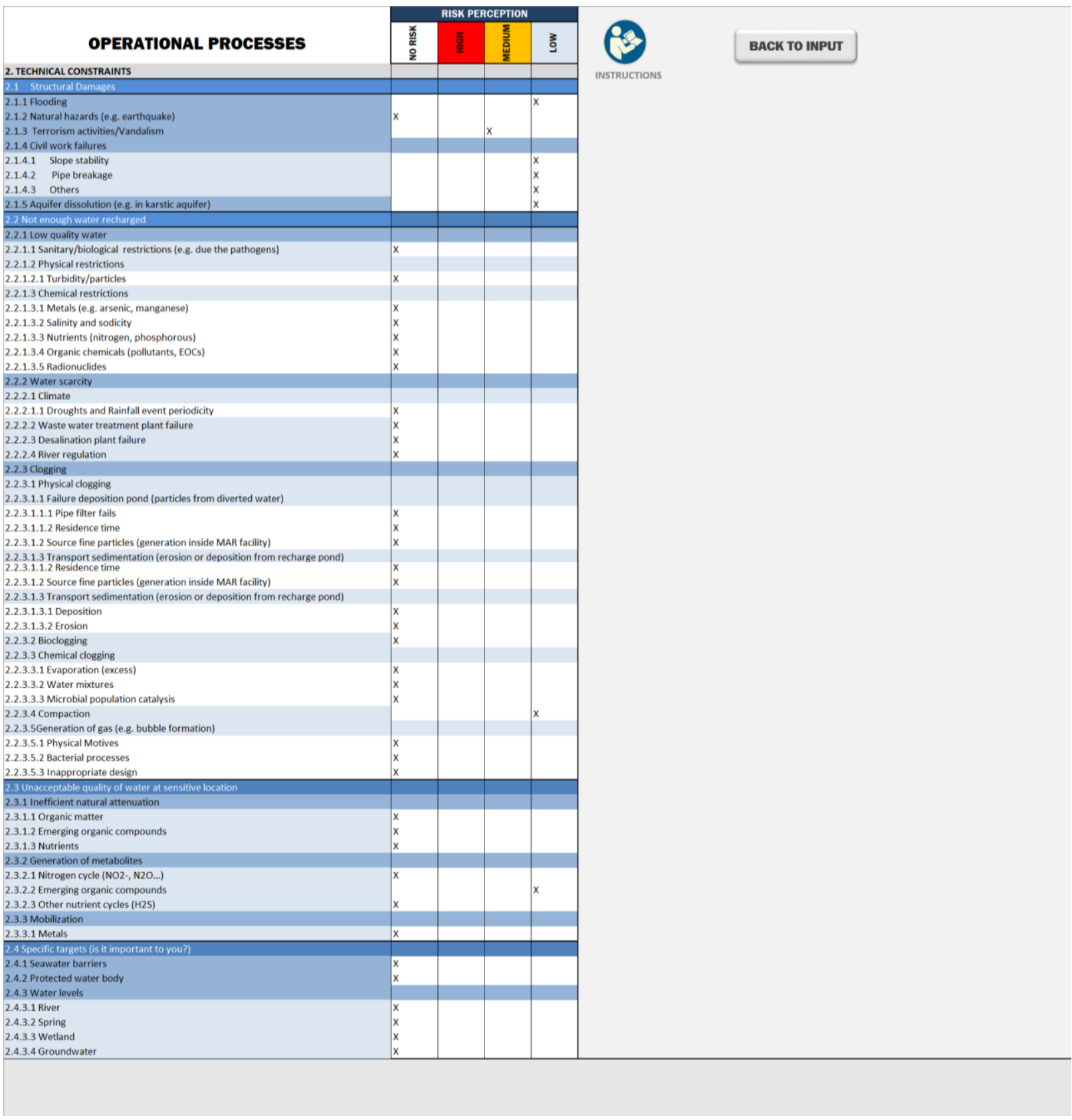

Figure S3.6. Technical constraints - Operation, sheet visualization.

The third step is RESULTS. This part shows the user the numerical results of the risk assessment (Figure S3.8). The risk assessment is calculated within the same Results sheet by applying the values present in the A PRIORI CRITERIA sheet (Figure S3.7) which are chosen depending on the risk category selected from the INPUT step. Note that the a priori criteria are site dependent. For that, the MAR facility managerd must define each a priori criteria based on their knowledge about the site and its particular 
idiosyncrasies. As a default, A PRIORI CRITERIA values are provided in MARRISKAPP based on experience from a number of sites worldwide. The a prior values are probability numbers (ranging in the interval [0-1] that indicate the probability (from a period of 2-6 years) that the MAR facility fails due to that particular individual event.
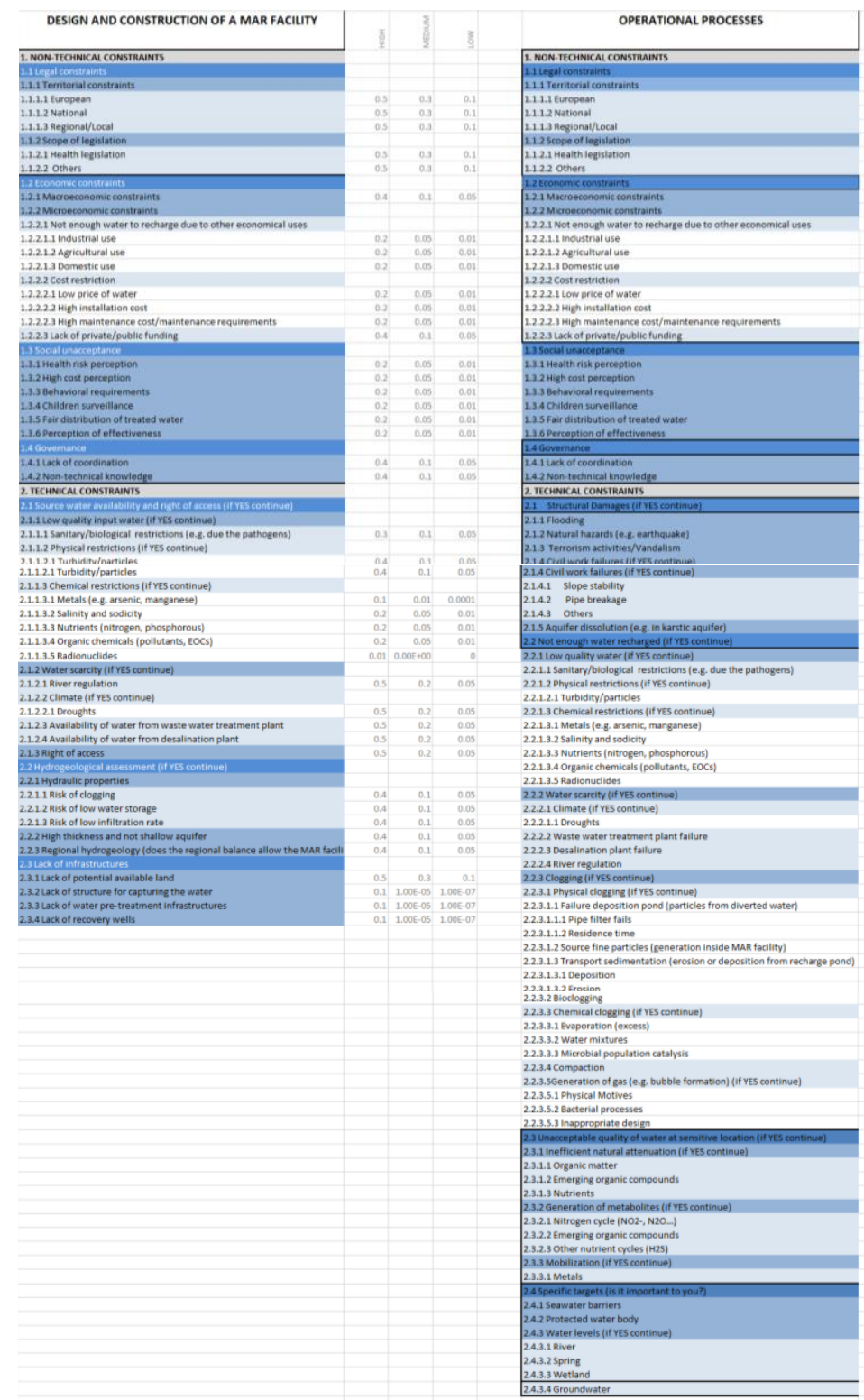

Figure S3.7. Expert Criteria values for the Llobregat site. 
The initial prior values are presented in the DEFAULT VALUE column, and are blocked to changes by the user. Next to this column, there is the CATEGORY DEFAULT VALUES column, which indicates the risk category that the user selected in the INPUT sheets. There is also a third column called USER VALUES, that can be modified by the user in order to change the specific risk values (from the DEFAULT VALUES column) if the user has better data than the default calculations for a specific study site (but does not want to change the a priori criteria values). This third column is the one that will be used in the following calculations, so the user has to be fully aware that its modification has direct consequences on the results. The tool indicates the user if these USER VALUES have been modified or not from the default ones (this is done by filling the USER VALUES cells with red color, to indicate that both columns have the same values). Similarly, to the other steps, a HELP button can be found, and also some instructions pop-up (Figure S3.9) if the instructions button is clicked. The user can change some data from the INPUT by clicking the BACK TO INPUT button. If everything is correct, the user can go to the next step by clicking the GRAPHICAL RESULTS button.

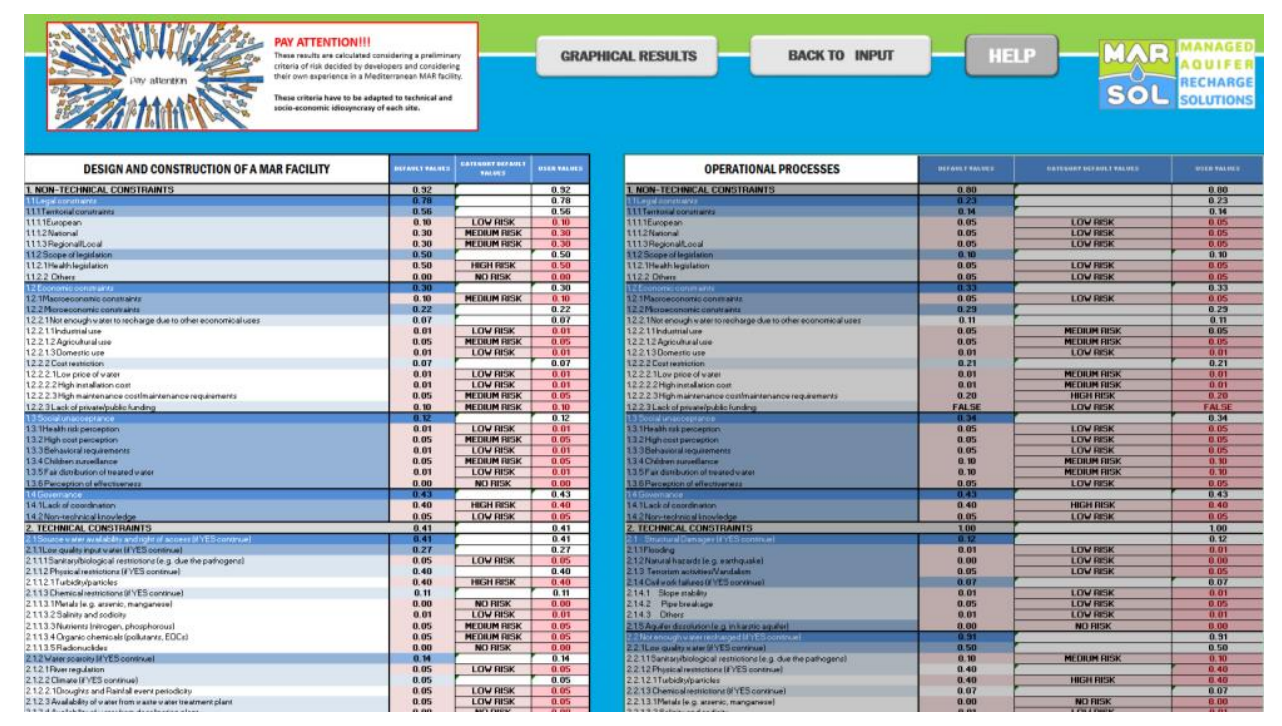

Figure S3.8. Results (upper part) sheet visualization. 


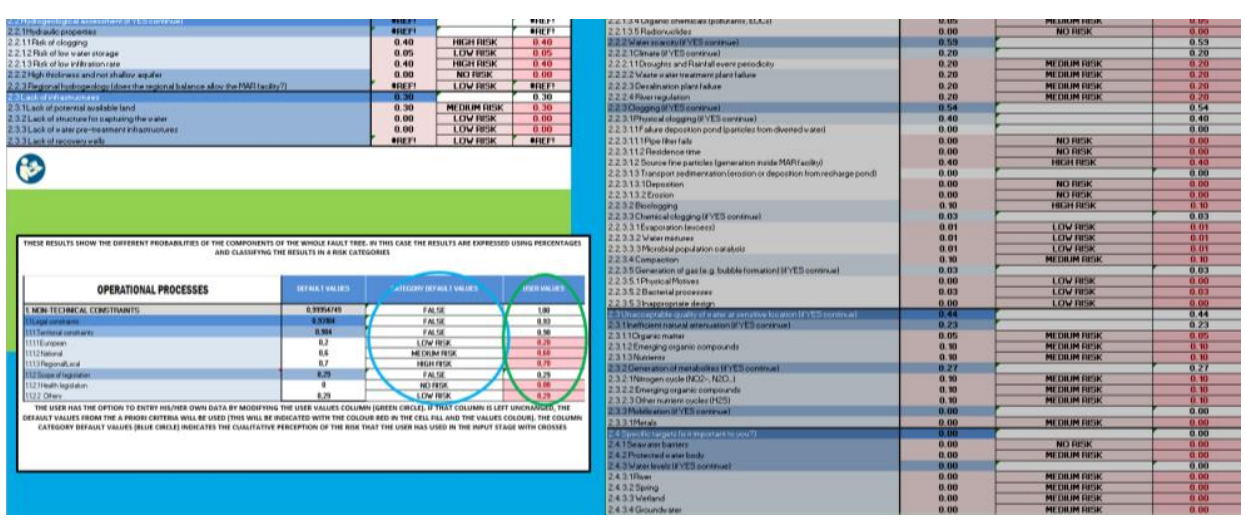

Figure S3.9. Results (bottom part) sheet visualization.

The fourth step of the MAR-RISKAPP is the graphical results (Figure S3.10), displaying the numerical results shown in the previous step into graphs and tables. This step is divided into four points: 1 ) Operational pivot-table results (Figure S3.11), 2) Design and construction pivot-table results (Figure S3.12), 3) Operational fault tree (Figure S3.13) and 4) Design and construction fault tree (Figure S3.14). As in the other steps, there is a button with instructions, only if the user needs some help or orientation with the results from this step. For both pivot-tables, the results are structured in four categories of risk (high, medium, low, and no-risk). Inside each category, risk values are displayed in decreasing order (from high to low risk values). Also, both pivottables have a button to go back to the graphical results main sheet. For both fault trees, each point from the survey is presented by using a rectangle. For each point, risk value is showed on the bottom-left part of the rectangle and also is colored according to risk categories. Finally, both fault-trees have a button to go back to the graphical results main sheet and a button to print the fault tree in a PDF file. 


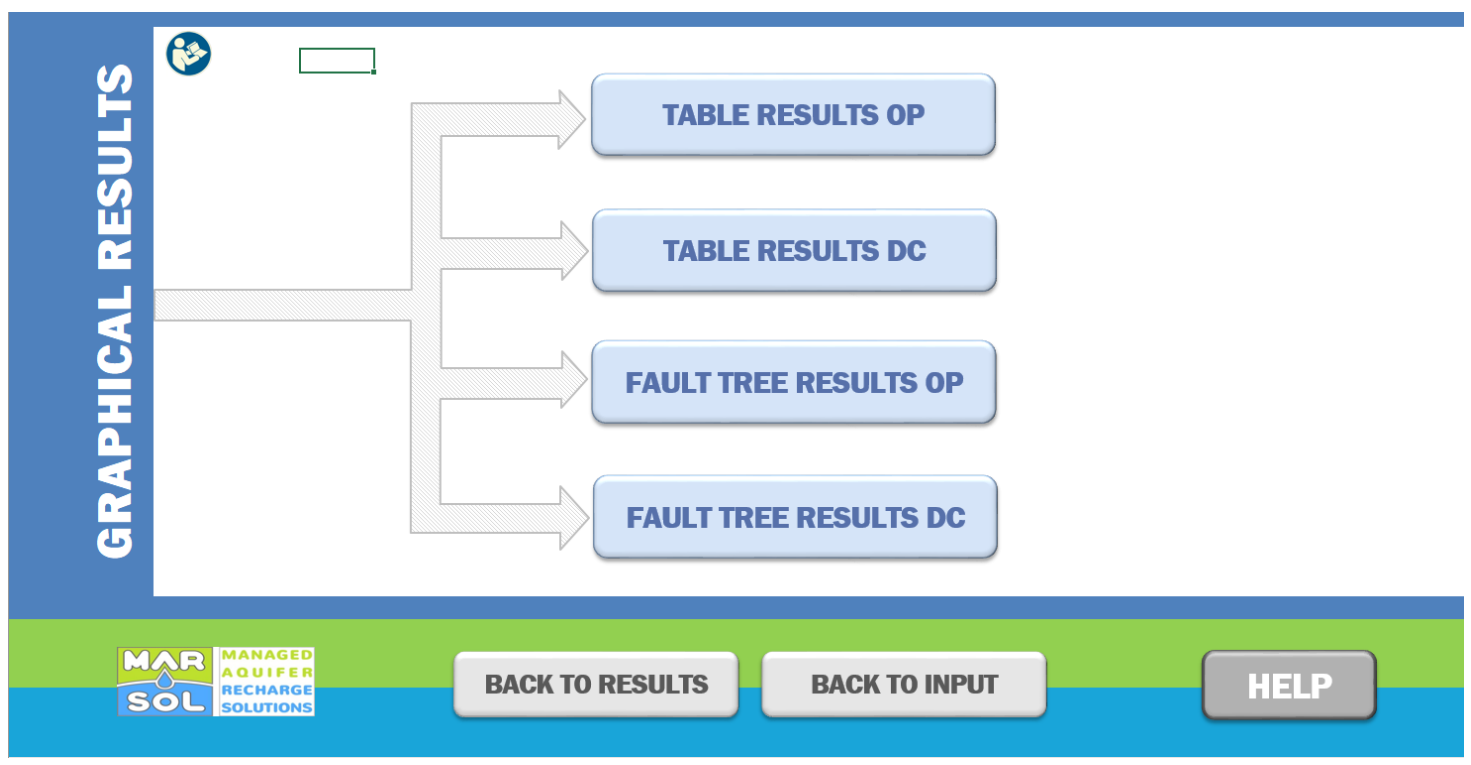

Figure S3.10. Graphical results sheet visualization.

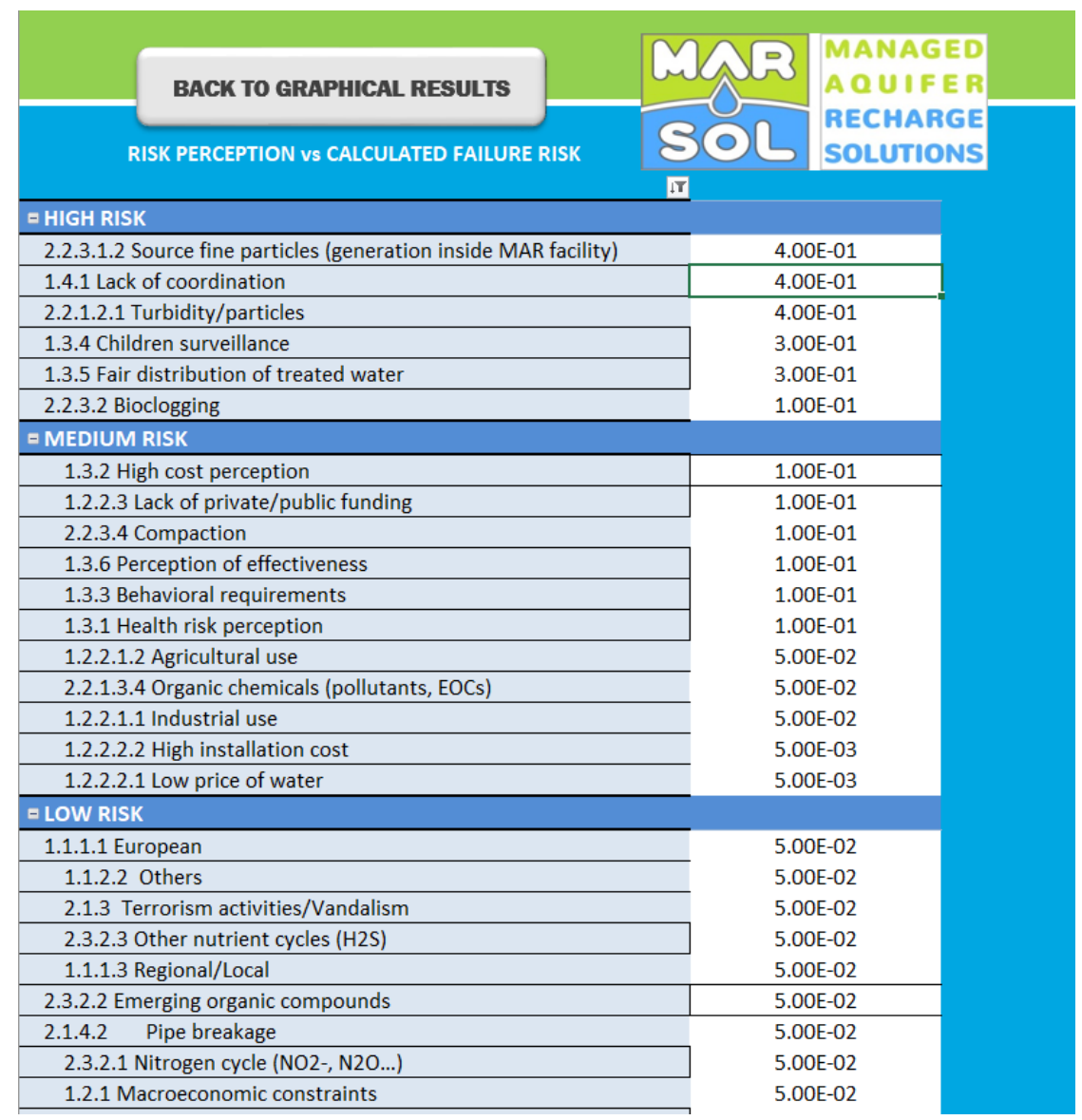

Figure S3.11. Operational pivot-table results sheet visualization. 


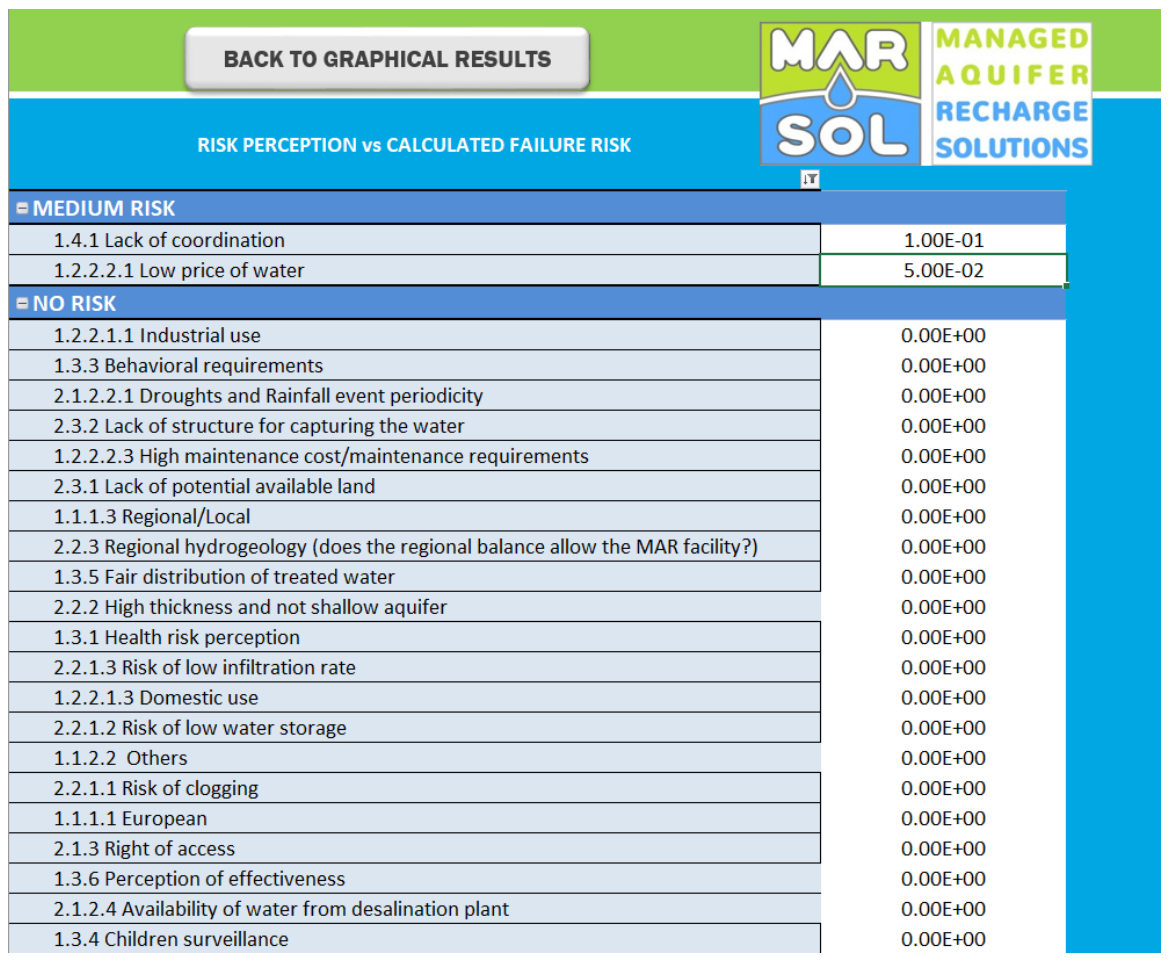

Figure S3.12. Design and construction pivot-table results sheet visualization. 


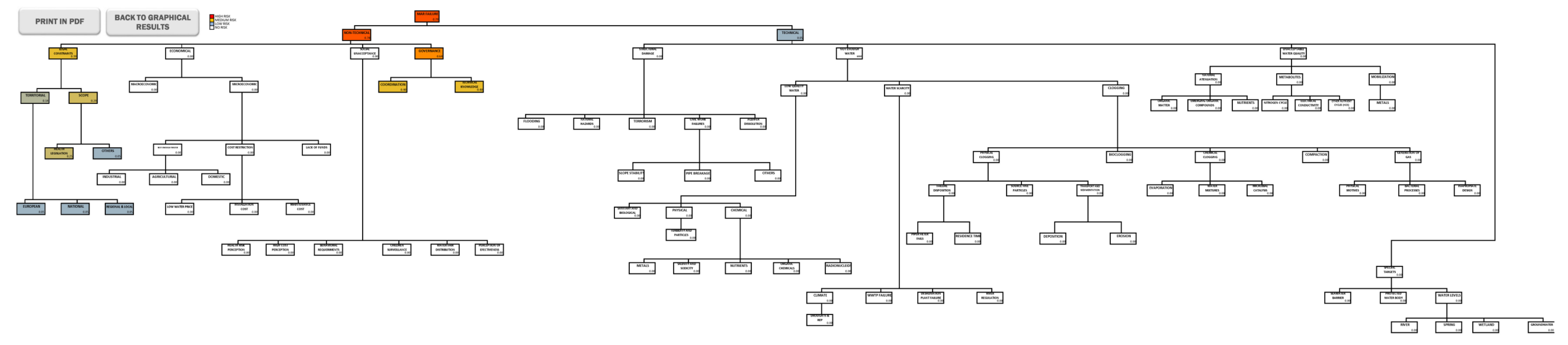

Figure S3.13. Operational fault-tree results sheet visualization.

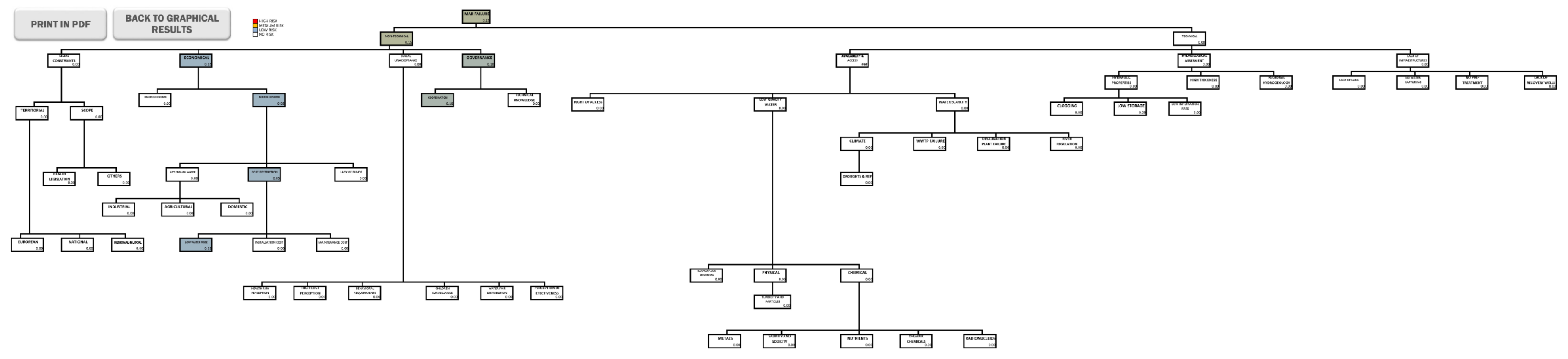

Figure S3.14. Design and construction sheet visualization. 
